\title{
Analog Self-Interference Cancellation Using Auxiliary Transmitter Considering IQ Imbalance and Amplifier Nonlinearity
}

\author{
Takahisa Fukui, Kazuki Komatsu, Student Member, IEEE, Yuichi Miyaji, Member, IEEE, and \\ Hideyuki Uehara, Member, IEEE
}

\begin{abstract}
In-band full-duplex communication, which transmits and receives simultaneously on the same frequency, causes self-interference (SI). In this paper, to cancel SI present in the radio frequency (RF) domain, we propose a novel nonlinear SI cancellation approach using an auxiliary transmitter which is effective in the presence of IQ imbalance and nonlinear distortion. The proposed approach estimates the local transceiver channel by using a time-domain least squares method and creates a signal for SI cancellation based on estimation results and a finite impulse response filter, whose coefficients are derived in this paper. Additionally, we theoretically calculate the SI cancellation limit of the proposed approach. Information about the SI cancellation limit due to phase noise is important for meeting SI cancellation requirements and being able to compare the effects of RF impairments such as IQ imbalance and nonlinear distortion. From simulation results, we show that the proposed approach outperforms the conventional approach and the case of using a general adaptive algorithm for the proposed approach. Furthermore, the SI cancellation limit is improved by adjusting the propagation delay of the SI signal and the canceling signal in addition to sharing one local oscillator in the local transceiver.
\end{abstract}

Index Terms-In-band full-duplex, self-interference, auxiliary transmitter, nonlinear, phase noise.

\section{INTRODUCTION}

I $\mathrm{N}$-band full-duplex (IBFD) communication, which transmits and receives simultaneously on the same frequency, can gain high throughput as compared to conventional wireless communication systems [1]. The application of IBFD to various communication systems, such as wireless local area networks (WLANs) [2], [3], device-to-device relay communications [4], [5], cellular networks [6], [7], and self-backhauling systems [8], has been studied. However, self-interference (SI) cancellation, where the requirement is generally in excess of $100 \mathrm{~dB}$, is still a major issue for realizing IBFD communication [9]-[11]. Furthermore, SI, which has a large power, causes the saturation of the low noise amplifier (LNA) and analog-to-digital converter (ADC) in the local receiver and reduces the allocated bits for the desired signal at the ADC. For this reason, SI cancellations in the propagation and/or analog domain are necessary [12].

This work was supported by Japan Society for the Promotion of Science (JSPS) KAKENHI Grant Numbers JP18K04138, JP19K14979, and JP19J12727.

T. Fukui, K. Komatsu, Y. Miyaji, and H. Uehara are with the Department of Electrical and Electronic Information Engineering, Toyohashi University of Technology, Japan (e-mail: fukui@comm.ee.tut.ac.jp; komatsu@comm.ee.tut.ac.jp; miyaji@ee.tut.ac.jp; huehara@ieee.org).
Propagation techniques that cancel SI in the propagation domain, such as passive approaches [13], active approaches [14], [15], and antenna interface approaches [16], [17], have been studied, and they aim to minimize the direct path coupling between both transmitter and receiver.

On the other hand, the main purpose of analog techniques that cancel SI in the analog domain is to cancel not only SI direct path components but also reflective path components. Analog techniques use two approaches. The first approach uses an analog circuit that utilizes the power amplifier (PA) output signal as the input signal to this cancellation circuit $[18]-[25]$. Generally, it consists of some digital controllers and passive elements such as splitters, variable attenuators, variable phase shifters, and delay lines. Using this approach, since the cancellation circuit estimates the SI channel between the radio frequency $(\mathrm{RF})$ front-end of the transmitter and receiver, the transceiver's RF impairments such as IQ imbalance, phase noise, and the PA nonlinear distortion can be ignored while canceling SI. However, according to the authors of [25], the components needed for attenuation and delay adjustment have nonlinearity and complicates subsequent SI cancellations. Furthermore, the required number of taps for sufficient SI cancellation and its optimization are still under investigation.

The second approach uses an auxiliary transmitter, which transmits the signal-canceling SI present in the RF domain [26]-[36]. In this approach, the canceling signal is digitally created based on the channel estimation. Unlike the analog circuit approach, this channel includes not only wireless channels between the RF front-end of the transmitter and receiver but also the channel between the auxiliary transmitter and receiver, and RF impairments. However, the auxiliary transmitter approach is flexible enough to handle multipath channels and wideband SI because the canceling signal is created in the digital domain. Furthermore, for multi-input and multi-output (MIMO) transceivers with $N_{\mathrm{TX}}$ transmit and $N_{\mathrm{RX}}$ receive antennas, while the analog circuit approach requires $N_{\mathrm{TX}} \times N_{\mathrm{RX}}$ cancellers, the auxiliary transmitter approach only requires $N_{\mathrm{RX}}$ cancellers to be implemented. Thus, the latter has a less complexity of the transceiver design than the former [35], 36].

After applying propagation techniques and/or analog techniques to prevent the receiver saturation, digital techniques, which have been studied [37]-[46], are generally used to cancel the digitalized SI in the final step of a series of cancellation processes. The digital techniques estimate the SI 
channel between the digital-to-analog converter (DAC) and $\mathrm{ADC}$, and the reproduced SI based on estimation results cancels SI in the digital domain. As previously mentioned, the requirement of SI cancellation is over $100 \mathrm{~dB}$ throughout the SI cancellation process including propagation, analog, and digital techniques. However, the transceiver noises such as thermal noise, phase noise, and quantization noise, restrict SI cancellation because they cannot be accurately estimated, and there are cases when the requirement of SI cancellation is not satisfied, namely, there is the SI cancellation limit due to the transceiver noise. In this paper, we describe this SI cancellation limit and the auxiliary transmitter approach.

\section{A. Review of Previous Research: Auxiliary Transmitter Ap- proach}

The authors of [29] have shown that the auxiliary transmitter approach can cancel wideband SI precisely and outperforms the analog circuit approach proposed by the authors of [25]. However, in [29], the transceiver's RF impairments, such as IQ imbalance and nonlinear distortion are not considered. The authors of [33] have proposed an auxiliary transmitter approach using a widely linear filter to generate the canceling signal that is effective in the presence of IQ imbalance. In [33], the proposed approach is robust against IQ imbalance, but its cancellation greatly decreases with increasing the PA nonlinear distortion.

In [34]-[36], the auxiliary transmitter approaches have been proposed in the presence of a PA nonlinear distortion without considering IQ imbalance. Generally, in order to estimate the PA nonlinear distortion, LNA nonlinear distortion should be avoided or separated from the PA nonlinear distortion. The authors of [34] have proposed a two-step approach where the PA nonlinear distortion and linear SI channel are estimated separately. In [34], the PA output is received by an auxiliary transceiver to avoid the LNA, and the auxiliary transceiver transmits the canceling signal generated based on the received PA nonlinear distortion and the estimated linear SI channel. In [35], the SI channel in the presence of the PA nonlinear distortion can be estimated by using least squares (LS) method. In [35], the proposed approach bypasses the LNA when sending the training signal to estimate the SI channel. By estimating the PA nonlinear distortion, both simulation and measurement results in [35] have demonstrated the excellent SI cancellation performance. Besides, in order to avoid bypassing LNA, the authors of [36] have proposed an auxiliary transmitter approach with an adaptive algorithm. In [36], the basis functions of the sent signal are orthogonalized with respect to each other, and the correlation between the nonlinear distortion of PA and LNA is minimized. Thereby, the proposed adaptive algorithm gains fast convergence and low mean square error.

Furthermore, in the conventional approaches of [34]-[36], the auxiliary transmitter approach has been implemented by adopting some commercial hardware. For example, the conventional approach of [36] has adopted LTE-Advanced Band 1 base station hardware and the Analog Devices evaluation board, which is equipped with two RF transmitter chains, to the measurement setup. As a result, the conventional approaches of [34]-[36] have shown the cancellation performance and have proved both feasibility and practicality of the auxiliary transmitter approach. However, these approaches cannot cancel SI to the noise floor defined by some additive noise, and both cancellation potential of the auxiliary transmitter approach and RF impairment effect restricting the cancellation performance cannot be identified precisely. Besides, in contrast to [33], IQ imbalance is not mentioned in [34]-[36].

When IQ imbalance is included, there are two effects. First, the training signal to estimate the SI channel is affected by the IQ imbalance at the receiver's IQ mixer, which cannot be bypassed. Generally, there is no need for channel estimation after the SI cancellation point where SI is combined with the canceling signal. Second, since both image and nonlinear components exist, the unknown coefficients that need to be estimated increases. In [37], [38], the unknown coefficients including both image and nonlinear components are estimated. However, since the proposed approaches in [37], [38] cancel SI in the digital domain, the cancellation points of these approaches and the auxiliary transmitter approach are different, and their corresponding signals for SI cancellation are also different.

Based on the above, we deal with both IQ imbalance and PA nonlinear distortion and analyze the auxiliary transmitter approach in this paper. These advantages are shown in Section I.C.

\section{B. Review of Previous Research: SI Cancellation Limit}

Even if the estimation for SI cancellation is perfect, the transceiver noises restrict SI cancellation, and there are cases when the requirement of SI cancellation is not satisfied. In particular, phase noise, which is one of the transceiver noises, is known to be the main SI cancellation limiting factor, and its effects on digital SI cancellation approach have been studied [39]-[45]. The authors of [43]-[45] have shown that phase noise can be suppressed by sharing a local oscillator (LO) between the transmitter and receiver. Thereby, the SI cancellation limit due to phase noise is greatly improved. Besides, sharing a LO, the authors of [43], [44] have theoretically calculated the phase noise power and the residual SI power as the SI cancellation limit of digital SI cancellation approach. On the other hand, the effects of phase noise in the auxiliary transmitter approach have not been well studied, and the SI cancellation limit is unknown. Knowing the SI cancellation limit due to phase noise is important for meeting SI cancellation requirements.

\section{Our Contribution}

As can be seen in Table 1 that shows the comparison of the auxiliary transmitter approaches in terms of addressed RF impairments and evaluation methods, the major novelties of our proposed approach are to enhance the addressed RF impairments to more cancel SI, and to newly evaluate the SI cancellation performance by the theoretical analysis. In the former, the enhancement of SI cancellation in the RF domain leads to omit the digital SI cancellation and the preprocessing 
TABLE I: Comparison of auxiliary transmitter approaches in terms of addressed RF impairments and evaluation methods. IQI, NL, PN, Impl., Sim., and Theo. mean IQ imbalance, nonlinear distortion, phase noise, implementation, simulation, theoretical analysis, respectively.

\begin{tabular}{|c|c|c|c|c|c|c|}
\hline \hline & \multicolumn{3}{|c|}{ Addressed RF impairments } & \multicolumn{3}{|c|}{ Evaluation methods } \\
\hline & IQI & NL & PN & Impl. & Sim. & Theo. \\
\hline & $\checkmark$ & & & & $\checkmark$ & \\
\hline Our proposed approach & $\checkmark$ & $\checkmark$ & $\checkmark$ & & $\checkmark$ & \\
\hline \hline 34 & & $\checkmark$ & & $\checkmark$ & $\checkmark$ & $\checkmark$ \\
\hline \hline
\end{tabular}

required for this in addition to getting more LNA gain and ADC bits. The preprocessing before the communication with other transceiver includes the channel estimation and both transmission and reception of the training signal for this. In the latter, our theoretical analysis can verify the auxiliary transmitter approach from a viewpoint different from the practical validation by the conventional approaches of [34]-[36] and can provide both cancellation potential of the auxiliary transmitter approach and RF impairment effect restricting the cancellation performance.

In order to achieve the optimum cancellation on the auxiliary transmitter approach under IQ imbalance, we need to apply the inverse of the auxiliary transmitter characteristics to the canceling signal, i.e., equalization of the auxiliary transmitter characteristics, because the canceling signal generated in the digital domain is affected by the auxiliary transmitter characteristics on its way to the SI cancellation point in the RF domain. Although the conventional approaches of [33][38] cannot cancel the SI signal affected by IQ imbalance and nonlinear distortion while equalizing the auxiliary transmitter characteristics where IQ imbalance exists, we propose the estimation and cancellation algorithm to achieve this. In the proposed algorithm, using the basis functions including both image and nonlinear components of the transmitted signal, the finite impulse response (FIR) filter is applied to each basis function, and we derive the optimum coefficients for this FIR filter. In addition, since the FIR filter coefficients include unknown IQ imbalance coefficients and nonlinear distortion coefficients, we show the estimation method of FIR filter coefficients by using time-domain LS method. As above, we work to maximize the cancellation performance of the auxiliary transmitter approach by using the digital signal processing.

Furthermore, since the analog techniques such as LO sharing and propagation delay operation are proposed in [45], [46], incorporating these analog techniques and our digital signal processing, we further theoretically analyze the SI cancellation limit to investigate the cancellation performance potential of the auxiliary transmitter approach. This analysis is similar to [43], which uses digital SI cancellation. However, unlike [43], this paper uses an auxiliary transmitter. For this, in this paper, there are three phase noises of the transmitter, receiver, and auxiliary transmitter, and their power values are calculated. Furthermore, unlike [43], we consider both IQ imbalance and nonlinear distortion. For this, we provide complex equation transformations that go back and forth in the space of time and frequency to calculate the residual SI power as shown in Appendix A. In addition, since the nonlinear distortion and transmitter IQ imbalance are included in the PA output signal, the PA output power is calculated by utilizing orthogonal frequency division multiplexing (OFDM) characteristics.

In this paper, a wide range of performance evaluation is performed by the SI cancellation limit analysis and numerical simulation. As a result, we show that the proposed approach can achieve higher SI cancellation than the conventional approach and the case of using a general adaptive algorithm for the proposed approach. In addition, we show that knowing the SI cancellation limit due to phase noise is important for not only meeting SI cancellation requirements but also comparing the effects of RF impairments.

The rest of this paper is organized as follows. Section II provides a detailed model of the SI, including IQ imbalance, phase noise, and nonlinear distortion, and mathematical operators, which represent the input-output characteristic of each component. The proposed approach is presented in Section III. In Section IV, the SI cancellation limit of the proposed approach is theoretically derived. In Section V, the performance of the proposed approach in equivalent baseband signal simulations and the SI cancellation limit are provided under different scenarios. Finally, the conclusions are presented in Section VI.

\section{SySTEM MODEL}

In this section, we describe the overall operation of the IBFD transceiver discussed in this paper. Figure 11 shows the IBFD transceiver model. The auxiliary transmitter creates and transmits the canceling signal $x_{\mathrm{aux}}[n]$, and the SI signal $x[n]$ is canceled in the RF domain. In this paper, the operation of the IBFD transceiver is divided into an estimation phase and a cancellation phase. In the estimation phase, the LNA is bypassed, as in [35], to estimate the local transceiver channel and avoid the nonlinear amplification at the LNA. Furthermore, we assume that the SI signal and canceling signal distortions at each component of the transceiver are represented by baseband equivalent models, and the desired signal is not considered to focus on SI cancellation.

Although this paper is based on the numerical simulation and theoretical analysis, we explain the perspective of a practical implementation. For Fig. 1, since the system of the auxiliary transmitter approach cancels SI by digital signal processing, it can basically be assembled without any special hardware. Besides, in Fig. 1. LO sharing is needed. However, 


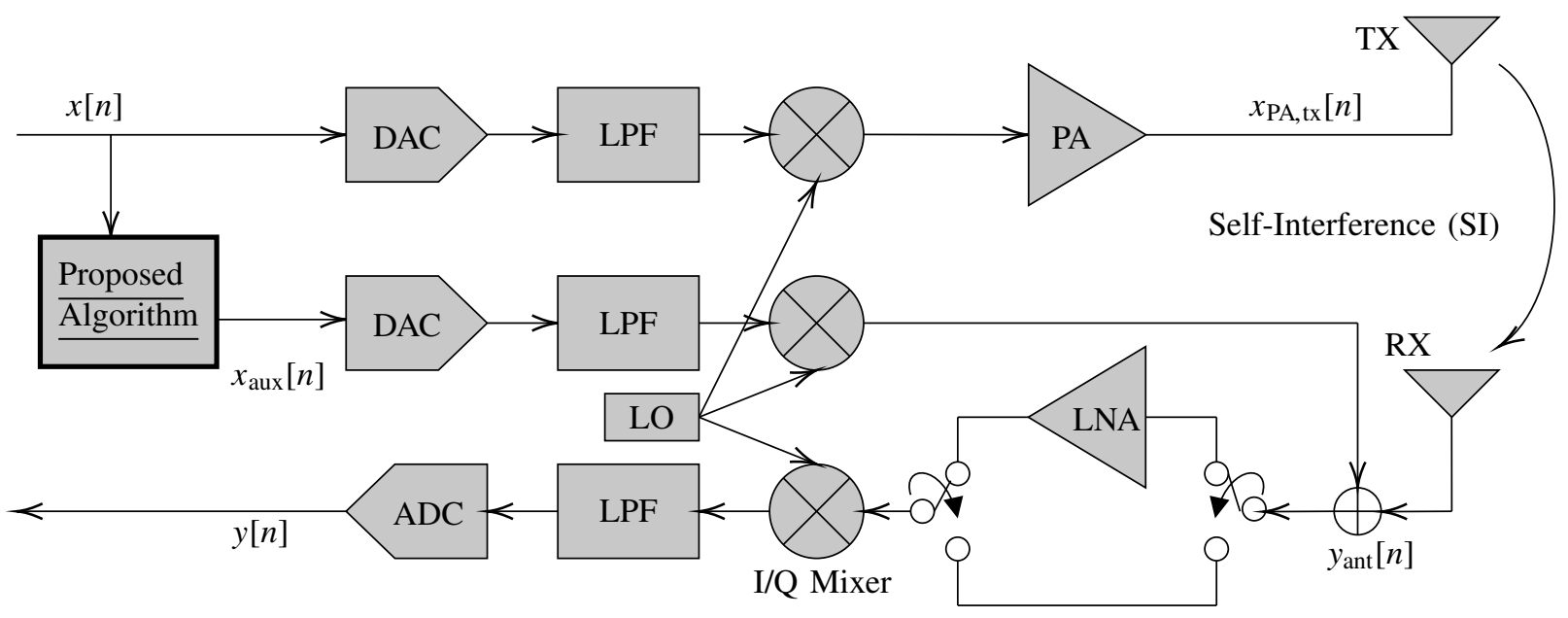

Fig. 1: A block diagram of the IBFD transceiver.

since the technique of LO sharing is implemented in [46], we believe that this technique can be incorporated into the auxiliary transmitter approach. Furthermore, when the proposed approach is extended to MIMO, the digital processing of each auxiliary transmitter is the same as single-input and singleoutput (SISO) system that is described in this paper. However, in order to cancel the phase noise of the SI signal by utilizing the phase noise of the canceling signal, the transmitter LO must be shared among all auxiliary transmitters.

\section{A. Transmitter}

1) Digital Domain: The transceiver transmits an OFDM signal that has $N$ subcarriers. In OFDM modulation, inverse discrete Fourier transformation (IDFT) is applied to the frequency-domain transmitted signal $X[k]$ where $k$ is the subcarrier index. Thereby, $X[k]$ is transformed to the timedomain signal $x[n]$ expressed as

$$
x[n]=\frac{1}{N} \sum_{k=0}^{N-1} X[k] e^{j \frac{2 \pi k n}{N}}
$$

where $n$ is the discrete time index. After IDFT, a cyclic prefix is added to the beginning of the time-domain transmitted signal $x[n]$.

2) IQ Mixer: The digital baseband signal $x[n]$ is converted to an analog baseband signal by the DAC and low path filter (LPF), and is then up-converted to an RF signal by the IQ mixer. On an ideal IQ mixer, the output equivalent baseband signal of the transmitter IQ mixer is equal to the input baseband signal. However, the IQ mixer has imbalances between the I and Q branches. The output signal of the transmitter IQ mixer can be expressed as [37], [38]

$$
x_{\mathrm{IQ}, \mathrm{tx}}[n]=x[n]+\eta_{\mathrm{tx}} x^{*}[n],
$$

where $\eta_{\mathrm{tx}}$ is the imbalance coefficient of the transmitter IQ mixer, and $(\cdot)^{*}$ denotes the complex-conjugate operation. $\eta_{\mathrm{tx}}$ quantifies the quality of the IQ mixer as image rejection ratio (IRR), and the transmitter IRR is defined as

$$
\operatorname{IRR}_{\mathrm{tx}}=\left|\eta_{\mathrm{tx}}\right|^{-2} \text {. }
$$

Phase noise $\phi(\cdot)$, which is generated at the LO, gives a random phase rotation to the output signal of the IQ mixer. The output signal affected by the transmitter phase noise is expressed as [43]

$$
x_{\mathrm{PN}, \mathrm{tx}}[n]=x_{\mathrm{IQ}, \mathrm{tx}}[n] e^{j \phi\left(n T_{s}\right)},
$$

where $T_{S}$ denotes the sampling interval. Both IQ imbalance and phase noise affect the output signal of the IQ mixer, and their order is (2) to (4) in the transmitter, i.e., phase noise after IQ imbalance [47], [48].

3) Power Amplifier: The output signal of the transmitter IQ mixer $x_{\mathrm{PN}, \mathrm{tx}}[n]$ is amplified by the PA of the transmitter for communication with a faraway terminal. Although PA enables high power transmission, the transmitted signal is affected by nonlinear distortion, which is called intermodulation distortion. Using the nonlinear function $f_{\mathrm{PA}}(\cdot)$ that is characterized by the AM/AM and AM/PM conversion, the PA output signal is expressed as [49, p. 732]

$$
x_{\mathrm{PA}, \mathrm{x}}[n]=f_{\mathrm{PA}}\left(x_{\mathrm{PN}, \mathrm{tx}}[n]\right) .
$$

Furthermore, the AM/AM and AM/PM conversion means that both amplitude and phase distortion of the PA output does not depend on the input phase. For this reason, the PA output is separated from the phase noise $\phi(\cdot)$ and can be rewritten as

$$
x_{\mathrm{PA}, \mathrm{tx}}[n]=\tilde{x}_{\mathrm{PA}, \mathrm{tx}}[n] e^{j \phi(n T s)},
$$

where

$$
\tilde{x}_{\mathrm{PA}, \mathrm{tx}}[n]=f_{\mathrm{PA}}\left(x_{\mathrm{IQ}, \mathrm{tx}}[n]\right) .
$$

4) Propagation Domain: The output signal of the PA is radiated from the transmitter antenna to the receiver antenna of the local transceiver as strong SI. The SI signal at the receiver antenna is expressed as

$$
y_{\mathrm{tx}}[n]=h_{\mathrm{tx}}[l] \otimes\left(\tilde{x}_{\mathrm{PA}, \mathrm{tx}}[n] e^{j \phi\left(n T_{s}-\tau_{\mathrm{tx}}\right)}\right),
$$

where $h_{\mathrm{tx}}[l]$ is the impulse response, $\tau_{\mathrm{tx}}$ is the propagation delay between the transmitter and receiver, and $\otimes$ denotes convolution. For notational convenience, the impulse response $h_{\mathrm{tx}}[l]$ models the joint effects of the PA memory effect, the 
wireless channel, and the phase rotation by $\tau_{\mathrm{tx}}$. Furthermore, the propagation delay $\tau_{\mathrm{tx}}$ can be less than the sampling interval $T_{s}$, which allows the phase noise $\phi\left(n T_{s}-\tau_{\mathrm{tx}}\right)$ to be expressed in detail.

\section{B. Auxiliary Transmitter}

The transceiver receives not only the SI signal $y_{\mathrm{tx}}[n]$ but also the canceling signal $y_{\text {aux }}[n]$ that is transmitted by the auxiliary transmitter to cancel the SI signal $y_{\mathrm{tx}}[n]$. In this paper, we assume that the wired channel $h_{\text {aux }}$ between the auxiliary transmitter and receiver is a single path, and the canceling signal $y_{\text {aux }}[n]$ at the receiver antenna is expressed as

$$
y_{\mathrm{aux}}[n]=h_{\mathrm{aux}}\left(x_{\mathrm{aux}}[n]+\eta_{\mathrm{aux}} x_{\mathrm{aux}}^{*}[n]\right) e^{j \phi\left(n T_{s}-\tau_{\mathrm{aux}}\right)},
$$

where $x_{\text {aux }}[n]$ is the transmitted signal of the auxiliary transmitter, $\eta_{\text {aux }}$ is the imbalance coefficient of the auxiliarytransmitter IQ mixer, and $\tau_{\text {aux }}$ is the propagation delay between the auxiliary transmitter and receiver. Here, since one LO is shared in the local transceiver, the phase noise $\phi(\cdot)$ is also common.

In the auxiliary transmitter approach, since the SI cancellation point exists in the RF domain, $x_{\text {aux }}[n]$ needs to cancel the SI signal $y_{\mathrm{tx}}[n]$ while equalizing the auxiliary transmitter channel. The purpose of this paper is how to generate $x_{\text {aux }}[n]$ to cancel SI and how to estimate the coefficients such as the impulse response and both image and nonlinear distortion. $x_{\text {aux }}[n]$ is made from the transmitted signal $x[n]$ through the proposed algorithm that is indicated in Section III.

\section{Receiver}

1) SI Cancellation Point: The SI signal cannot be recorded in RF domain and is canceled in real time at the combiner attached to the receiver, i.e., the SI cancellation point. The received signal at the SI cancellation point is expressed as

$$
y_{\text {ant }}[n]=y_{\mathrm{tx}}[n]+y_{\mathrm{aux}}[n]+z[n],
$$

where $z[n]$ is an additive white Gaussian noise (AWGN).

2) Low Noise Amplifier: Generally, the received signal is amplified by the LNA because the desired signal from a faraway terminal is greatly attenuated. Although the desired signal is not considered in the system model, actually, the received signal contains the desired signal when canceling the SI signal (cancellation phase), thus the LNA is necessary to amplify the received signal including the desired signal. The LNA output is expressed as

$$
y_{\mathrm{LNA}}[n]=f_{\mathrm{LNA}}\left(y_{\mathrm{ant}}[n]\right),
$$

where $f_{\mathrm{LNA}}(\cdot)$ is a nonlinear function that is characterized by the AM/AM and AM/PM conversion [49, p. 732]. However, since preventing nonlinear amplification at the LNA is one of the purposes of the auxiliary transmitter approach, the LNA can be operated in the linear region if SI cancellation is achieved. In addition, when sending the training signal for the LS method that estimates the local transceiver channel (estimation phase), the LNA is bypassed as in [35] to avoid its nonlinear amplification, i.e., $y_{\mathrm{LNA}}[n]=y_{\text {ant }}[n]$.

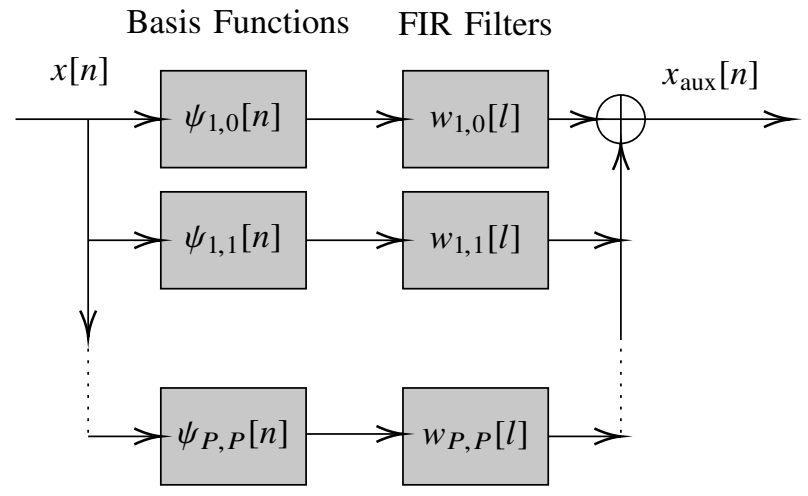

Fig. 2: Block diagram of the canceling signal generation in the proposed algorithm.

3) IQ Mixer: The output signal of the LNA is downconverted to an analog baseband signal by the receiver IQ mixer. At this time, both IQ imbalance and phase noise occur at the receiver IQ mixer. The received signal affected by the receiver phase noise is expressed as

$$
y_{\mathrm{PN}}[n]=y_{\mathrm{LNA}}[n] e^{-j \phi\left(n T_{s}\right)} .
$$

In down-conversion, the receiver phase noise has the opposite sign to the transmitter phase noise [41]-[43]. Although the LO output is input to two IQ mixers in [46], the auxiliary approach have three IQ mixers. However, it has barely effect on the SI cancellation performance that the $\mathrm{LO}$ of the receiver is not shared. This is because the receiver IQ mixer exists after the SI cancellation point, and the phase noise of the receiver does not affect the SI cancellation performance.

Finally, the received signal is affected by the receiver IQ imbalance and is expressed as

$$
y[n]=y_{\mathrm{PN}}[n]+\eta_{\mathrm{rx}} y_{\mathrm{PN}}^{*}[n],
$$

where $\eta_{\mathrm{rx}}$ is the imbalance coefficient of the receiver IQ mixer. As with the transmitter, both IQ imbalance and phase noise affect the output signal of the IQ mixer, but in the receiver, their order is in contrast to the transmitter, i.e., IQ imbalance after phase noise [47], [48].

\section{PRoposed Approach}

Figure 2 shows the proposed algorithm for generating the canceling signal $x_{\mathrm{aux}}[n]$ from the transmitted signal $x[n]$. In order to achieve the optimum cancellation in the auxiliary transmitter approach under IQ imbalance, we need to apply the inverse of the auxiliary transmitter characteristics to the canceling signal, i.e., equalization of the auxiliary transmitter characteristics, because the canceling signal generated in the digital domain is affected by the auxiliary transmitter characteristics on its way to the SI cancellation point in the RF domain. However, the FIR filters designed by [35], [36] cannot equalize the auxiliary transmitter characteristics with IQ imbalance and cancel the image components of the SI signal. Furthermore, in the literature of digital SI cancellation, there is no equalization of the auxiliary transmitter characteristics because it cancels SI in the digital domain, not the RF domain. 
For this reason, the FIR filters designed by [37], [38] also cannot be applied to the system in this paper. Therefore, we first derive the optimum FIR filter coefficients $w_{p, q}[l]$ in Section III.A to enable that $x_{\text {aux }}[n]$ cancels SI in the RF domain. Next, we show the proposed algorithm using the time-domain LS method and derived FIR filter coefficients in Section III.B.

\section{A. Derivation of Optimum Filter Coefficients}

In the proposed approach, the PA output signal is approximated by a parallel Hammerstein as in [38] and is expressed as 1

$$
\begin{aligned}
x_{\mathrm{PA}, \mathrm{tx}}[n] & \approx \sum_{p=1,3, \ldots}^{\infty} a_{p} x_{\mathrm{IQ}, \mathrm{tx}}[n]\left|x_{\mathrm{IQ}, \mathrm{tx}}[n]\right|^{p-1} \\
& =\sum_{p=1,3, \ldots}^{\infty} \sum_{q=0}^{p} a_{p} c_{q, p-q} x^{q}[n]\left(x^{*}[n]\right)^{p-q},
\end{aligned}
$$

where $a_{p}$ is the $p$-th nonlinear distortion coefficient, and $c_{q, p-q}$ is the coefficient including the transmitter IQ imbalance coefficient $\eta_{\mathrm{tx}}$. Actually, although $a_{p}$ includes the transmitter phase noise, we ignore this in the proposed algorithm, i.e., $\phi(\cdot) \approx 0$. For simplicity, we define this as:

$$
\psi_{p, q}[n]=x^{q}[n]\left(x^{*}[n]\right)^{p-q} .
$$

From (14) and (15), in the cancellation phase, the received signal of (13) can be rewritten as

$$
\begin{aligned}
y[n]= & g_{\mathrm{LNA}}\left\{\sum_{p=1,3, \ldots}^{P} \sum_{q=0}^{p}\left\{d_{p, q}[l] \otimes \psi_{p, q}[n]\right\}\right. \\
& \left.+b_{1} x_{\mathrm{aux}}[n]+b_{2} x_{\mathrm{aux}}^{*}[n]\right\}+z_{\mathrm{all}}[n],
\end{aligned}
$$

where

$$
\begin{aligned}
d_{p, q}[l] & =\alpha_{p} c_{q, p-q} h_{\mathrm{tx}}[l]+\eta_{\mathrm{rx}} \alpha_{p} c_{q, p-q}^{*} h_{\mathrm{tx}}^{*}[l], \\
b_{1} & =h_{\mathrm{aux}}+\eta_{\mathrm{rx}} \eta_{\mathrm{aux}}^{*} h_{\mathrm{aux}}^{*}, \\
b_{2} & =\eta_{\mathrm{aux}} h_{\mathrm{aux}}+\eta_{\mathrm{rx}} h_{\mathrm{aux}}^{*} .
\end{aligned}
$$

Both $d_{p, q}[l]$ and $b_{(\cdot)}$ are combined unknown coefficients, and $P$ is the maximum order to estimate distortions. Furthermore, $z_{\text {all }}[n]$ is all additive noise including $z[n]$ and the nonlinear distortion of the order after $P$-th. Since we focus on the cancellation phase in Section III.A, the LNA is not bypassed. However, in the auxiliary transmitter approach, the SI signal is canceled in the RF domain, and it can be regarded that the power of the received signal after the SI cancellation is small enough not to be nonlinearly amplified at the LNA. For this reason, we approximate the LNA as the linear model with the gain $g_{\text {LNA }}$.

Focusing on (16), due to the presence of IQ imbalance in the channel of the auxiliary transmitter side, the canceling signal $x_{\text {aux }}[n]$ needs to equalize this channel. Furthermore, in order to cancel the SI signal, the basis functions of the canceling

1 We show this derivation as a supplemental information along with the simulation code of Section $\mathrm{V}$ in our GitHub page (https://github.com/TakahisaFukui/Analog_Self_Interference_Cancellation _Using_Auxiliary_Transmitter_Considering_IQI_and_NL). signal $x_{\text {aux }}[n]$ must be matched to the SI signal including both image and nonlinear components. In the proposed approach, the canceling signal is created as

$$
x_{\text {aux }}[n]=\sum_{p=1,3, \ldots}^{P} \sum_{q=0}^{p} w_{p, q}[l] \otimes \psi_{p, q}[n],
$$

where $w_{p, q}[l]$ are FIR filter coefficients for each basis function. From [20, the received signal in 16 can be rewritten as

$$
\begin{gathered}
y[n]=g_{\mathrm{LNA}} \sum_{p=1,3, \ldots}^{P} \sum_{q=0}^{p}\left\{d_{p, q}[l]+b_{1} w_{p, q}[l]+b_{2} w_{q, p}^{*}[l]\right\} \\
\otimes \psi_{p, q}[n]+z_{\mathrm{all}}[n] .
\end{gathered}
$$

From 21, when the SI signal can be completely canceled, i.e., $y[n]=z_{\text {all }}[n]$, we can obtain two equations as follows:

$$
\begin{aligned}
& d_{p, q}[l]+b_{1} w_{p, q}[l]+b_{2} w_{q, p}^{*}[l]=0, \\
& d_{q, p}[l]+b_{1} w_{q, p}[l]+b_{2} w_{p, q}^{*}[l]=0 .
\end{aligned}
$$

By solving 222 and 23, we can obtain the filter coefficients $w_{p, q}[l]$, and they are expressed as

$$
w_{p, q}[l]=\frac{b_{1}^{*} d_{p, q}[l]-b_{2} d_{q, p}^{*}[l]}{\left|b_{2}\right|^{2}-\left|b_{1}\right|^{2}} .
$$

In [24), $w_{p, q}[l]$ indicates the optimum filter coefficients, and if $d_{p, q}[l]$ and $b_{(\cdot)}$ are known, the SI signal can be completely canceled. Here, note that both unknown coefficients $d_{p, q}[l]$ and $b_{(\cdot)}$ include the imbalance coefficient of the receiver IQ mixer $\eta_{\mathrm{rx}}$. For this reason, it seems that the condition equations (22) and (23) are satisfied at the receiver IQ mixer, and the SI signal before SI cancellation is input to the LNA. However, that is not right, and the condition equations are satisfied at the SI cancellation point. From (17), (18), and (19), the filter coefficients can be expanded as

$$
w_{p, q}[l]=-\frac{\alpha_{p} c_{p, p-q} h_{\mathrm{aux}}^{*} h_{\mathrm{tx}}[l]-\eta_{\mathrm{aux}} \alpha_{p} c_{q, p-q}^{*} h_{\mathrm{aux}} h_{\mathrm{tx}}^{*}[l]}{\left|h_{\mathrm{aux}}\right|^{2}\left(1-\left|\eta_{\mathrm{aux}}\right|^{2}\right)} .
$$

Focusing on $[25]$, the filter coefficients $w_{p, q}[l]$ do not include $\eta_{\mathrm{rx}}$, and the condition $y[n]=z_{\mathrm{all}}[n]$ does not depend on the receiver IQ imbalance. Thus, before the receiver IQ mixer, namely at the SI cancellation point, the SI signal can be completely canceled when $d_{p, q}[l]$ and $b_{(\cdot)}$ are known.

\section{B. Proposed Algorithm}

In Section III.A, we derive the optimum FIR filter coefficients for SI cancellation, but it is necessary to estimate the unknown coefficients $d_{p, q}[l]$ and $b_{(\cdot)}$ to use them. In the proposed algorithm, both unknown coefficients $d_{p, q}[l]$ and $b_{(\cdot)}$ are estimated by using the time-domain LS method. These estimated coefficients $\hat{d}_{p, q}[l]$ and $\hat{b}_{(\cdot)}$ are substituted into the optimum filter coefficients as derived in 24, and the canceling signal $x_{\text {aux }}[n]$ is generated in 20$]$. In the estimation phase, the received signal $y^{\prime}[n]$ is expressed as

$$
\begin{aligned}
y^{\prime}[n]=\sum_{p=1,3, \ldots}^{P} \sum_{q=0}^{p}\left\{d_{p, q}[l] \otimes \psi_{p, q}[n]\right\} \\
+b_{1} x_{\text {aux }}[n]+b_{2} x_{\text {aux }}^{*}[n]+z_{\text {all }}[n] .
\end{aligned}
$$


Since the LNA is bypassed in the estimation phase, $\sqrt{26}$ is the same as (16) except for $g_{\mathrm{LNA}}$. From (26), when $M$ samples are observed for the LS, the received signal vector $\mathbf{y}$ is represented as

$$
\begin{aligned}
\mathbf{y} & =\mathbf{X h}+\mathbf{z}, \\
\mathbf{y} & =\left[y^{\prime}[n], \ldots, y^{\prime}[n+M-1]\right]^{T}, \\
\mathbf{h} & =\left[d_{1,0}[0], \ldots, d_{1,0}[L-1], d_{1,1}[0], \ldots, b_{1}, b_{2}\right]^{T}, \\
\mathbf{z} & =\left[z_{\text {all }}[n], \ldots, z_{\text {all }}[n+M-1]\right]^{T},
\end{aligned}
$$

where $L$ is the length of the impulse response $h_{\mathrm{tx}}[l]$, and $\mathbf{X}$ is shown in (31).

From (27), the LS minimizes the sum of squared residuals $\|\mathbf{y}-\mathbf{X h}\|^{2}$, and the unknown coefficients can be estimated as

$$
\begin{aligned}
& \hat{\mathbf{h}}=\underset{\mathbf{h}}{\arg \min }\|\mathbf{y}-\mathbf{X h}\|^{2}=\left(\mathbf{X}^{H} \mathbf{X}\right)^{-1} \mathbf{X}^{H} \mathbf{y}, \\
& \hat{\mathbf{h}}=\left[\hat{d}_{1,0}[0], \ldots, \hat{d}_{1,0}[L-1], \hat{d}_{1,1}[0], \ldots, \hat{b}_{1}, \hat{b}_{2}\right]^{T} .
\end{aligned}
$$

In the proposed algorithm, substituting these estimated coefficients for the FIR filter coefficients of (24), the canceling signal $x_{\text {aux }}[n]$ can be generated as shown Fig. 2

In terms of practical implementation of the auxiliary transmitter approach, according to [36], the PA nonlinearity and coupling channel change slowly, so that the estimation may be performed periodically offline. In addition, the period of the estimation can be reduced by reducing the computational complexity of the estimation and the number of the samples for the estimation. This estimation period is an overhead that does not exist in half-duplex communication, and reducing the estimation period is one of the issues in auxiliary transmitter approaches and digital SI cancellation approaches.

\section{Analysis of SElF-InTERFERENCE CANCELlation LIMIT}

Although the requirement of SI cancellation exceeds 100 $\mathrm{dB}$, the transceiver noise that cannot be accurately estimated restricts SI cancellation. Thereby, even if the estimation of the image and nonlinear components is perfect, there are cases when the requirement of SI cancellation is not satisfied, namely, there is an SI cancellation limit due to the transceiver noise.

In this section, we analyze the SI cancellation limit of the approach proposed in Section III. First, we calculate the residual SI signal after SI cancellation when the parallel Hammerstein model in (14) is completely expressed as the PA output signal, and when the estimation in (32) is correct, i.e., $\hat{\mathbf{h}}=\mathbf{h}$. In the cancellation phase, the residual SI signal at the SI cancellation point is expressed as

$$
\begin{aligned}
\hat{y}_{\mathrm{ant}}[n]= & h_{\mathrm{tx}}[l] \otimes\left(\tilde{x}_{\mathrm{PA}, \mathrm{tx}}[n] e^{j \phi\left(n T_{s}-\tau_{\mathrm{tx}}\right)}\right) \\
& \quad-\left(h_{\mathrm{tx}}[l] \otimes \tilde{x}_{\mathrm{PA}, \mathrm{tx}}[n]\right) e^{j \phi\left(n T_{s}-\tau_{\mathrm{aux}}\right)}+z[n] \\
= & \sum_{l=0}^{L-1} h_{\mathrm{tx}}[l] \tilde{x}_{\mathrm{PA}, \mathrm{tx}}[n-l] \\
& \times\left\{e^{j \phi\left((n-l) T_{s}-\tau_{\mathrm{tx}}\right)}-e^{j \phi\left(n T_{s}-\tau_{\mathrm{aux}}\right)}\right\}+z[n] .
\end{aligned}
$$

In (34), the first term is the SI signal, and the second term is the canceling signal. The SI signal is affected by the impulse response $h_{\mathrm{tx}}[l]$ after the transmitter phase noise. However, the canceling signal reproduces $h_{\mathrm{tx}}[l]$ in the digital domain, and then the auxiliary-transmitter phase noise affects the canceling signal.

From (35), the residual SI signal affected by the receiver phase noise $\hat{y}_{\mathrm{PN}}[n]$ is represented as

$$
\begin{aligned}
\hat{y}_{\mathrm{PN}}[n] & =g_{\mathrm{LNA}} \hat{y}_{\mathrm{ant}}[n] e^{-j \phi\left(n T_{s}\right)} \\
& =g_{\mathrm{LNA}}\left\{\sum_{l=0}^{L-1} h_{\mathrm{tx}}[l] \tilde{x}_{\mathrm{PA}, \mathrm{tx}}[n-l] u_{l}[n]+z[n] e^{-j \phi\left(n T_{s}\right)}\right\},
\end{aligned}
$$

where

$$
u_{l}[n]=\left\{e^{j \phi\left((n-l) T_{s}-\tau_{\mathrm{tx}}\right)}-e^{j \phi\left(n T_{s}-\tau_{\text {aux }}\right)}\right\} e^{-j \phi\left(n T_{s}\right)} .
$$

In the cancellation phase, it can be regarded that the received signal after SI cancellation in the RF domain is small enough not to be nonlinearly amplified at the LNA, so that the LNA can be expressed as the linear model with the gain $g_{\mathrm{LNA}}$.

From (36), the residual SI signal affected by the receiver IQ imbalance $\hat{y}[n]$ is represented as

$$
\hat{y}[n]=\hat{y}_{\mathrm{PN}}[n]+\eta_{\mathrm{rx}} \hat{y}_{\mathrm{PN}}^{*}[n] .
$$

Expression expansions up to (38) is a preparation for the analysis, and from here, we analyze the SI cancellation limit through calculating the residual SI power. From (38), the residual SI power can be derived as

$$
\mathbb{E}\left[|\hat{y}[n]|^{2}\right]=\left(1+\left|\eta_{\mathrm{rx}}\right|^{2}\right) \mathbb{E}\left[\left|\hat{y}_{\mathrm{PN}}[n]\right|^{2}\right],
$$

where $\mathbb{E}[\cdot]$ denotes the statistical expectation operator. The derivation of 39 is shown in Appendix A.

From (39), the residual SI power affected by the receiver phase noise $\mathbb{E}\left[\left|\hat{y}_{\mathrm{PN}}[n]\right|^{2}\right]$ can be derived as

$$
\begin{aligned}
& \mathbb{E}\left[\left|\hat{y}_{\mathrm{PN}}[n]\right|^{2}\right] \\
= & g_{\mathrm{LNA}}^{2} \mathbb{E}\left[\left|\sum_{l=0}^{L-1} h_{\mathrm{tx}}[l] \tilde{x}_{\mathrm{PA}, \mathrm{tx}}[n-l] u_{l}[n]+z[n] e^{-j \phi\left(n T_{s}\right)}\right|^{2}\right]
\end{aligned}
$$

where $\sigma_{z}^{2}=\mathbb{E}\left[|z[n]|^{2}\right]$. Here, the impulse response $h_{\mathrm{tx}}[l]$ that generally has fading, the PA output signal $\tilde{x}_{\mathrm{PA}, \mathrm{tx}}[n]$, and the combined phase noise $u_{l}[n]$ can be assumed to be independent of each other. For this, 40 is rewritten as

$$
\begin{aligned}
& \mathbb{E}\left[\left|\hat{y}_{\mathrm{PN}}[n]\right|^{2}\right] \\
=g_{\mathrm{LNA}}^{2} & \sum_{l=0}^{L-1}\left\{\mathbb{E}\left[\left|h_{\mathrm{tx}}[l]\right|^{2}\right] \mathbb{E}\left[\left|\tilde{x}_{\mathrm{PA}, \mathrm{tx}}[n-l]\right|^{2}\right]\right. \\
& \left.\times \mathbb{E}\left[\left|u_{l}[n]\right|^{2}\right]\right\}+g_{\mathrm{LNA}}^{2} \sigma_{z}^{2},
\end{aligned}
$$

From (41), the phase noise power is generally not large, but is multiplied by the SI power, which has a large power. For this reason, the effect of the phase noise on the residual SI power becomes relatively large.

In order to derive the PA output power $\mathbb{E}\left[\left|\tilde{x}_{\mathrm{PA}, \mathrm{tx}}[n]\right|^{2}\right]$, we utilize that the OFDM signal $x[n]$ can be expressed as a complex Gaussian random variable with zero mean and 


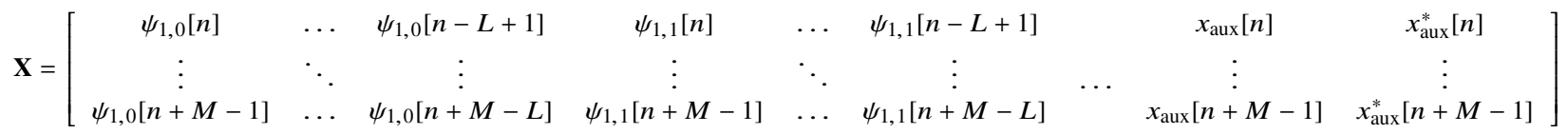

variance $\sigma^{2}$ due to the central limit theorem [50]. For this, the statistical expectation of the PA output power can be expressed as

$$
\begin{aligned}
\mathbb{E}\left[\left|\tilde{x}_{\mathrm{PA}, \mathrm{tx}}[n]\right|^{2}\right] & =\int_{-\infty}^{\infty} \int_{-\infty}^{\infty}\left|f\left(x[n]+\eta_{\mathrm{tx}} x^{*}[n]\right)\right|^{2} \frac{1}{\pi \sigma^{2}} \\
& \times \sigma_{\mathrm{PA}, \mathrm{tx}}^{2} .
\end{aligned}
$$

Here, since the solution of (42) depends on the arbitrary nonlinear function $f_{\mathrm{PA}}(\cdot)$, we calculate from (42) to (43) by using Simpson's rule, which is a method for numerical integration.

In order to derive the combined phase noise power $\mathbb{E}\left[\left|u_{l}[n]\right|^{2}\right]$, we need to determine the model of the LO type. In this analysis and the performance evaluation in Section V, we assume using free-running oscillator, whose phase noise can be modeled by a Wiener process, and it is defined as

$$
\phi(t)-\phi(t+\tau)=\Phi(\tau) \sim \mathcal{N}(0,4 \pi \beta \tau),
$$

where $\beta$ denotes the $3 \mathrm{~dB}$ bandwidth of the phase noise Lorentzian spectrum, $\tau$ is the time between the instants, and the phase variation $\Phi(\tau)$ is a Gaussian random variable with zero mean and variance $4 \pi \beta \tau[41]-[43],[51]$. By the way, note that although free-running oscillator is used in this paper, results of the performance evaluation are generally applicable. This is because common phase error (CPE) can be reduced when sharing LO, and the effect of the phase noise on SI cancellation closely resembles the practical phase-locked loop (PLL) based oscillators [43].

When using the free-running oscillator, the combined phase noise power can be derived in Appendix B, and is expressed as

$$
\mathbb{E}\left[\left|u_{l}[n]\right|^{2}\right]=2-2 e^{-2 \pi \beta\left|l T_{s}+\tau_{\mathrm{tx}}-\tau_{\mathrm{aux}}\right|} .
$$

In the direct path $(l=0)$, the combined phase noise power decreases as the propagation delay difference between $\tau_{\mathrm{tx}}$ and $\tau_{\text {aux }}$ decreases. Furthermore, even if $l \rightarrow \infty$, the combined phase noise $\mathbb{E}\left[\left|u_{l \rightarrow \infty}[n]\right|^{2}\right]$ does not continue to increase, and depends on the first term in (45). Namely, it doubles the SI power in the worst case.

From 41, 43, and 45, we can obtain the residual SI power of (39), and can calculate the SI cancellation limit defined as

$$
\text { SI Cancellation Limit }=\frac{\sigma_{\mathrm{PA}, \mathrm{tx}}^{2} \sum_{l=0}^{L-1} \mathbb{E}\left[\left|h_{\mathrm{tx}}[l]\right|^{2}\right]}{\mathbb{E}\left[|\hat{y}[n]|^{2}\right]},
$$

where the numerator denotes the SI power before the SI cancellation point.

\section{Performance Evaluation}

In this section, numerical simulation results with the SI cancellation limit are provided to validate the proposed approach. To show the effectiveness of the proposed approach, we compare the performance of the proposed approach with the conventional approaches.

\section{A. Simulation Environment}

To verify the proposed approach, equivalent baseband simulations of the IBFD transceiver shown in Fig. 1 are performed. In the simulation, unless otherwise specified, we use the parameters shown in TABLE $\mathrm{II}$.

In the proposed approach, the oversampling rate is set to 8 in order to estimate the nonlinear distortion up to the 7 th order. Besides, our IBFD transceiver of Fig. 1 has two antennas (one TX and one RX), and a direct component of the SI signal reaches between the antennas. Therefore, in the performance evaluation, the SI wireless channel is simulated as a Rician fading channel with a power delay profile where indirect signals decay by $20 \mathrm{~dB}$ at 1 sample. The Rician fading can be described by two parameters $K$ and $\Omega$. $K$ is the ratio of the power of the direct path to the power of the indirect path, and $\Omega$ is the total power of both direct and indirect paths. By using both parameters $K$ and $\Omega$, the Rician distribution is expressed as $C \mathcal{N}\left(\mu_{\mathrm{R}}, \sigma_{\mathrm{R}}^{2}\right)$, where $\mu_{\mathrm{R}}=\sqrt{K \Omega /(1+K)}$, and $\sigma_{\mathrm{R}}^{2}=\Omega /(1+K)$ [52]. Here, we define the following: $K_{\mathrm{dB}}=10 \log _{10}(K)=30 \mathrm{~dB}$ as in [33], [40], and $\Omega$ depends on SI-to-noise ratio (INR).

In the performance evaluation, both input-output characteristics of the PA and LNA are represented by Rapp model [53], which characterizes the AM/AM conversion of a solid-state high power amplifier. The Rapp model is expressed as

$$
f(x[n])=\frac{g x[n]}{\left\{1+\left(\frac{g|x[n]|}{V_{\text {sat }}}\right)^{2 s}\right\}^{\frac{1}{2 s}}},
$$

where $V_{\text {sat }}, g$, and $s$ are the saturation voltage, the small signal gain, and the smoothness factor, respectively. Furthermore, we assume that the $3 \mathrm{~dB}$ bandwidth of the phase noise is $50 \mathrm{~Hz}$ [43], and $\operatorname{IRR}_{\mathrm{dB}}=10 \log _{10}(\mathrm{IRR})=30 \mathrm{~dB}$ [37]. This IRR value is the same for the transmitter, receiver, and auxiliary transmitter.

\section{B. Results and Discussions}

Figure 3 shows both simulated (Sim.) and theoretical (Theo.) values of the SI cancellation limit (SICL), and the performances of the proposed approach when the maximum nonlinear order $P$ is less than 7 . We compare the proposed approach with the conventional approach proposed in [35] at $P=5$. As the conditions in Fig. 3. the number of OFDM symbols is 500, and the input backoff of PA is 
TABLE II: Specification

\begin{tabular}{c|c}
\hline Parameter & value \\
\hline Modulation & OFDM (16QAM) \\
\hline FFT size & 64 samples \\
\hline Oversampling rate & 8 \\
\hline \# of active subcarriers & 52 samples \\
\hline Cyclic prefix & 128 samples \\
\hline \# of OFDM symbols & 100 symbols \\
\hline Sampling rate & $20 \mathrm{MHz}$ \\
\hline SI wireless channel & Rician fading \\
\hline$K_{\mathrm{dB}}$ factor & $30 \mathrm{~dB}$ \\
\hline SI channel length & $4 \mathrm{samples}$ \\
\hline Propagation delay btw Aux-TX and $\mathrm{RX} \tau_{\mathrm{aux}}$ & $2 \mathrm{~ns}$ \\
\hline IRR $\mathrm{dB}$ & $1 \mathrm{~ns}$ \\
\hline Propation delay btw TX and $\mathrm{RX} \tau_{\mathrm{tx}}$ & $30 \mathrm{~dB}$ \\
\hline PA/LNA maximum input power & $50 \mathrm{~Hz}$ \\
\hline PA/LNA smoothness factor & $0 \mathrm{dBm}$ \\
\hline Input backoff & 3 \\
\hline AWGN power & $10 \mathrm{~dB}$ \\
\hline INR & $-100 \mathrm{dBm}$ \\
\hline \# of trials & $80 \mathrm{~dB}$ \\
\hline
\end{tabular}

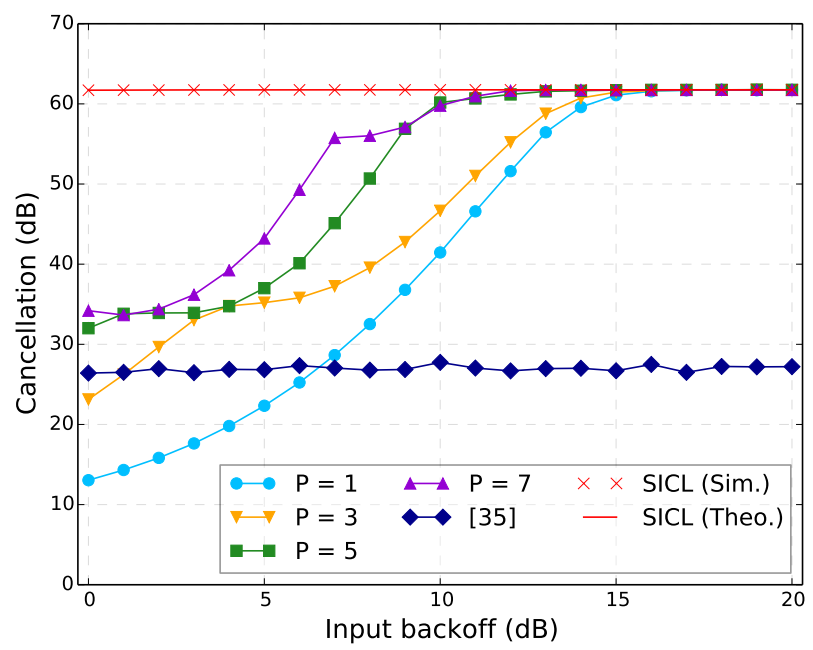

Fig. 3: SICL and the SI cancellation performances of both proposed and conventional approaches when the number of OFDM symbols is 500 and the input backoff of PA is varied.

varied. First, since the simulated and theoretical values of SICL are matched, we can theoretically and accurately obtain the achievable SI cancellation amount by (46). While the conventional approach deals with PA nonlinear amplification, the proposed approach deals with IQ imbalance in addition to this and achieves high cancellation performance. In Fig. 3 . the cancellation performance of the conventional approach is constantly about $27 \mathrm{~dB}$. In the simulation, IRR is set to 30 $\mathrm{dB}$, and image components that are $30 \mathrm{~dB}$ smaller than the linear component occur at the IQ mixers of the transmitter, receiver, and auxiliary transmitter. As a result, since the sum of the image components included in the received SI signal has $27 \mathrm{~dB}$ less power than the linear component, the conventional approach can cancel only $27 \mathrm{~dB}$. Meanwhile, the cancellation performance of the proposed approach is about $23 \mathrm{~dB}$ better than the conventional approach when the backoff is $10 \mathrm{~dB}$. This is because the proposed approach can cancel the SI signal affected by both IQ imbalance and PA nonlinear amplification while equalizing the auxiliary transmitter characteristics where IQ imbalance exists. However, when the backoff decreases, namely when the nonlinearity becomes strong, the proposed approach degrades due to the approximation of the PA output signal by the parallel Hammerstein in (14). Furthermore, if the order $P$ is increased, higher-order nonlinear components can be canceled, so that the cancellation performance increases. However, we can see that there are some backoff values where the performance is not improved even if the order $P$ is increased. For example, in the proposed approach, the performances at $P=5$ and $P=7$ are coincided when the backoff is more than $9 \mathrm{~dB}$, and the performances at $P=3$ and $P=5$ are coincided when the backoff is $4 \mathrm{~dB}$. This is because the nonlinear distortion power of lower order is not always larger than higher order's one [50]. In other words, the 7th order distortion power is lower than the 9th or higher order distortion power when the backoff is $9 \mathrm{~dB}$, and the 5th order distortion power is lower than the 7 th or higher order distortion power when the backoff is $4 \mathrm{~dB}$. In Fig. 3, the proposed approach achieves SICL when the backoff is more than $12 \mathrm{~dB}$. Focusing on SICL, it is constantly about $62 \mathrm{~dB}$ because the phase noise restricts the cancellation performance. Thus, the cancellation performance of the proposed approach at $P=5$ depends on the effect of the phase noise when the backoff is more than $12 \mathrm{~dB}$, and depends on the effect of the nonlinear distortion when the backoff is less than $12 \mathrm{~dB}$. Here, the proposed approach at $P=1$ means that the widely linear filter is adapted to the canceling signal of 20] as in [33]. The proposed approach at $P=1$ is vulnerable to nonlinear distortion, but it outperforms the conventional approach when the backoff is more than $7 \mathrm{~dB}$ and achieves SICL when the backoff is more than $16 \mathrm{~dB}$. For simplicity, since the proposed approaches with $P=7$ and 5 are equivalent when the backoff is more than $9 \mathrm{~dB}$, subsequent results do not show the proposed approach at $P=7$.

Figure 4 shows SICL and the SI cancellation performances of both proposed and conventional approaches when INR is varied. First, the proposed approaches at $P=1$ and 5 outperform the conventional approach at $P=5$ when INR is more than $20 \mathrm{~dB}$. In addition, both SICL and the proposed approach at $P=5$ are equal to INR when INR is less than 50 $\mathrm{dB}$. This is because the effects of the nonlinear distortion and phase noise are smaller than AWGN when INR is less than 50 $\mathrm{dB}$. Therefore, the proposed approach can completely cancel SI to the AWGN level when INR is less than $50 \mathrm{~dB}$. Besides, when INR is between $50 \mathrm{~dB}$ and $60 \mathrm{~dB}$, since the proposed approach and SICL have the same cancellation performance, it is found that the phase noise restricts the cancellation performance of the proposed approach. In addition, when INR is more than $60 \mathrm{~dB}$, since the proposed approach and SICL do not have the same cancellation performance, it is found that the nonlinear distortion restricts the cancellation performance of the proposed approach. For this, knowing SICL is important for not only meeting SI cancellation requirements but also comparing the effects of RF impairments. In Fig. 4 the 


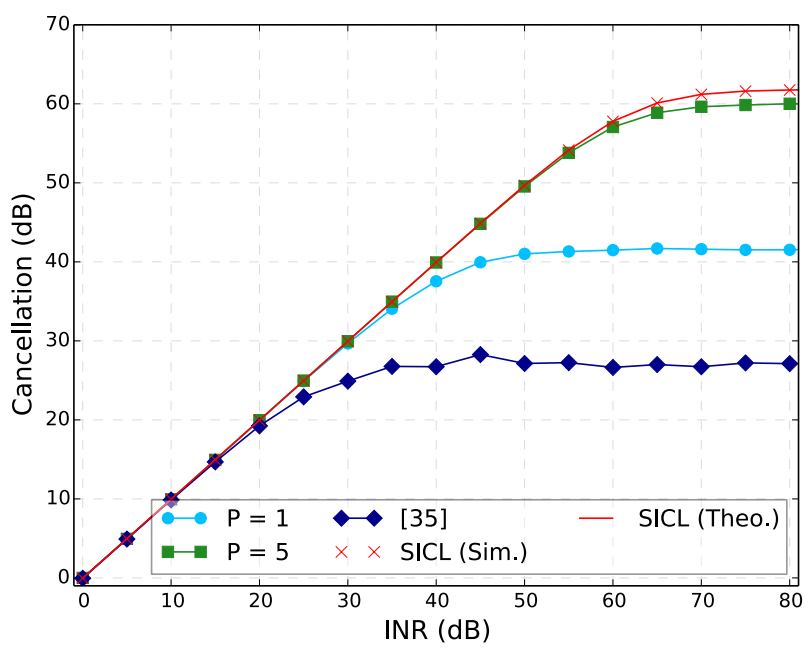

Fig. 4: SICL and the SI cancellation performances of both proposed and conventional approaches when INR is varied.

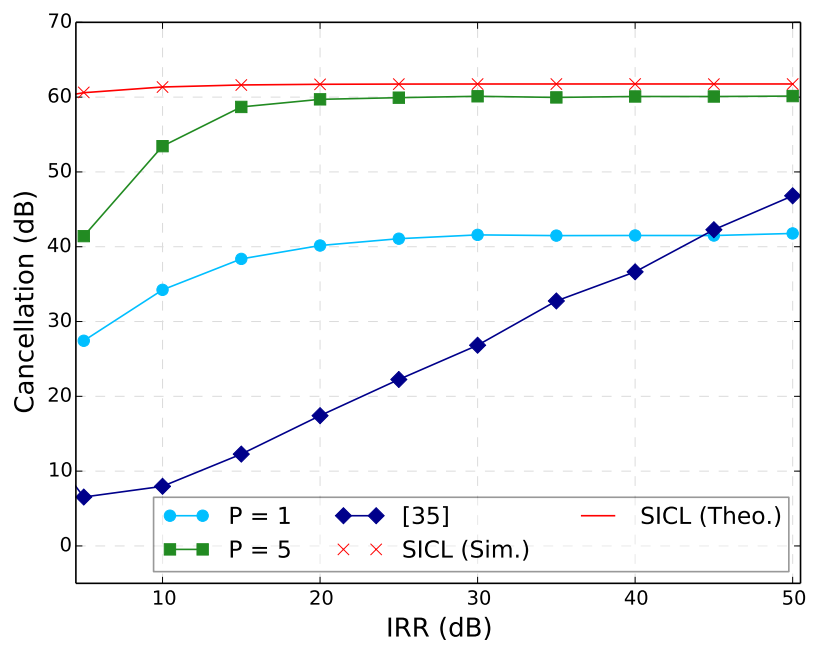

Fig. 5: SICL and the SI cancellation performances of both proposed and conventional approaches when IRR is varied.

cancellation performances of the conventional approach at $P=5$ and proposed approach at $P=1$ are up to $27 \mathrm{~dB}$ and $41 \mathrm{~dB}$, respectively. In the former, due to non-canceled image components, and in the latter, due to non-canceled nonlinear distortion, these performances are saturated. This result can be seen in Fig. 3 when the backoff is $10 \mathrm{~dB}$, which value is set as shown in Table $\Pi$

Figure 5 shows SICL and the SI cancellation performances of both proposed and conventional approaches when IRR is varied. According to [37], even the IRR of a high-quality IQ mixer is $35 \mathrm{~dB}$, but in this paper, we vary the IRR widely to show the trend of the SI cancellation performances. In Fig. 5. the conventional approach at $P=5$ improves as the IRR increases and outperforms the proposed approach at $P=1$ when IRR is more than $45 \mathrm{~dB}$. Furthermore, since this cancellation performance roughly increases as much as the

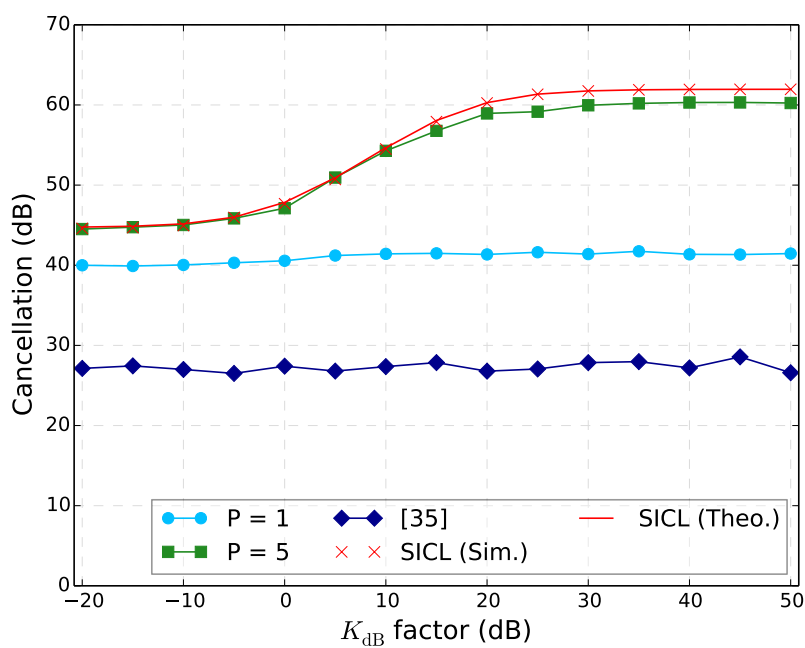

Fig. 6: SICL and the SI cancellation performances of both proposed and conventional approaches when $K_{\mathrm{dB}}$ factor is varied.

increase amount of IRR, it can be found that the conventional approach at $P=5$ is restricted by the image components that occur at the IQ mixers of the transmitter, receiver, and auxiliary transmitter. Meanwhile, the proposed approach at $P=5$ outperforms the conventional approach under all conditions in Fig. 5 because the proposed approach considers both IQ imbalance and nonlinear distortion. However, the proposed approach at $P=5$ does not reach SICL even if IRR increases. This is because, as can be seen from Fig. 3. there is the residual nonlinear distortion that cannot be represented by the parallel Hammerstein model with $P=5$. Furthermore, this residual nonlinear distortion include not only the linear component but also the image component. This is because the input signal of the PA is affected by the transmitter IQ imbalance. The degradation of the proposed approach when IRR is less than $15 \mathrm{~dB}$ is because the residual nonlinear distortion including the image component becomes larger than the residual nonlinear distortion including the linear component.

Figure 6 shows SICL and the SI cancellation performances of both proposed and conventional approaches when the $K_{\mathrm{dB}}$ factor of the Rician fading is varied. In Fig. 6, SICL and the proposed approach at $P=5$ degrades when $K_{\mathrm{dB}}$ is less than $25 \mathrm{~dB}$. This is because the indirect components $(l \neq 0)$ of the combined phase noise power in (45) is larger than its direct component $(l=0)$, and the effect of the indirect components increases as $K_{\mathrm{dB}}$ decreases. Furthermore, although SI, which propagates over a short distance, has a strong direct component, the situation when $K_{\mathrm{dB}} \leq 0 \mathrm{~dB}$ means that the direct component is less than and the indirect components. This situation is the same as [31]. In [31], both analog circuit and auxiliary transmitter approaches are utilized to cancel both direct and indirect components of SI, respectively. When $K_{\mathrm{dB}}=30 \mathrm{~dB}$, the proposed approach at $P=5$ achieves about $60 \mathrm{~dB}$ cancellation. If $K_{\mathrm{dB}}$ factor could be suppressed from $30 \mathrm{~dB}$ to $0 \mathrm{~dB}$ by the antenna isolation, the sum of 


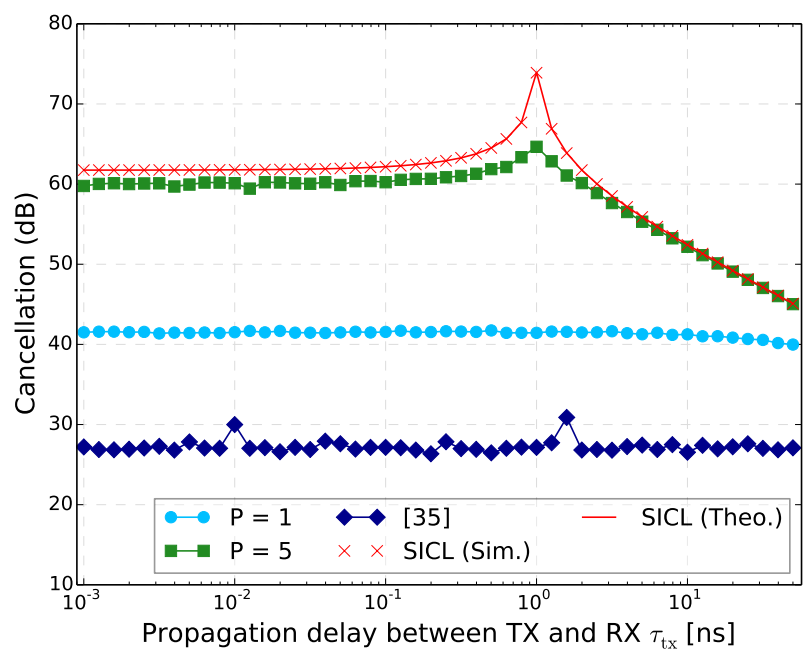

Fig. 7: SICL and the SI cancellation performances of both proposed and conventional approaches when the propagation delay between the transmitter and receiver $\tau_{\mathrm{tx}}$ is varied.

this suppression and the cancellation of the proposed approach when $K_{\mathrm{dB}}=0 \mathrm{~dB}$ is about $77 \mathrm{~dB}$. Therefore, it is effective to combine the techniques that increase the suppression of the wireless SI channel. Furthermore, when $K_{\mathrm{dB}} \leq 10 \mathrm{~dB}$, the performance of the proposed approach at $P=5$ is equal to SICL, and it can be found that the phase noise has the most influence among RF impairments.

Figure 7] shows SICL and the SI cancellation performances of both proposed and conventional approaches when the propagation delay between the transmitter and receiver $\tau_{\mathrm{tx}}$ is varied. In Fig.7. SICL is about $75 \mathrm{~dB}$ when $\tau_{\mathrm{tx}}=1 \mathrm{~ns}$. This is because the direct component $(l=0)$ of the combined phase noise power in (45) becomes zero when $\tau_{\mathrm{tx}}=\tau_{\mathrm{aux}}$. As with SICL, the proposed approach at $P=5$ has the maximum performance when $\tau_{\mathrm{tx}}=1 \mathrm{~ns}$, and its cancellation performance is about $65 \mathrm{~dB}$. The results show that, in addition to LO sharing, it is effective for SI cancellation that the propagation delay between the local transceiver is adjusted as in [45], [46]. For example, in the transmitter or auxiliary transmitter, a delay line is equipped after IQ mixer or between the LO and IQ mixer. Meanwhile, as the propagation delay difference between $\tau_{\mathrm{tx}}$ and $\tau_{\text {aux }}$ increases, SICL and the cancellation performance of the proposed approach at $P=5$ decrease exponentially. This is because, as can be seen from (45), the direct component of the combined phase noise power changes exponentially with respect to $\left|\tau_{\mathrm{tx}}-\tau_{\text {aux }}\right|$.

Figure 8 shows SICL and the SI cancellation performances of both proposed and conventional approaches when the 3 $\mathrm{dB}$ bandwidth of the phase noise is varied. In Fig. 8. SICL increases as the $3 \mathrm{~dB}$ bandwidth decreases, and its maximum value is $80 \mathrm{~dB}$ because the effect of the phase noise becomes lower than AWGN. Furthermore, when the $3 \mathrm{~dB}$ bandwidth is more than $1 \mathrm{~Hz}$, SICL exponentially degrades, and this trend is the same as [43], whose approach cancels SI present in the digital domain. As well as SICL, in the proposed approach at

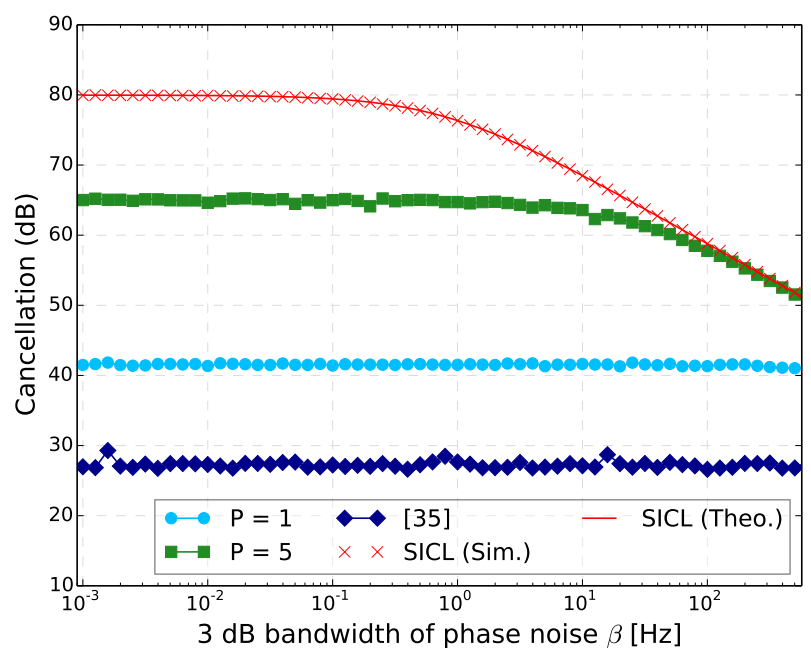

Fig. 8: SICL and the SI cancellation performances of both proposed and conventional approaches when the $3 \mathrm{~dB}$ bandwidth of the phase noise is varied.

$P=5$, the cancellation performance is improved as the $3 \mathrm{~dB}$ bandwidth decreases, and its maximum value is about $65 \mathrm{~dB}$. For this reason, the $3 \mathrm{~dB}$ bandwidth of the phase noise should be as small as possible to cancel SI.

\section{Comparison of Estimation Algorithms}

Here, we compare both cancellation performances and computational complexities of the proposed algorithm and conventional algorithms of [33], [35], [36]. In [33], [36], least mean square (LMS), which is one of the adaptive algorithms, is adopted to estimate the FIR filters that are used to generate the canceling signal of the auxiliary transmitter. In this paper, these conventional algorithms are executed in the simulation environment shown in Table [I Since the basis functions are orthogonalized with respect to each other in [36], we realize this orthogonalization through the singular value decomposition described in [54], as with [36]. In addition, we also show the result that the FIR filter of (24) is estimated by the adaptive algorithm. Although the basis functions are normalized in [36], in the proposed approach, the power of the basis functions is different from each other, so that normalized least mean square (NLMS) is used in the proposed approach.

Table III shows both cancellation performances and computational complexities. Step sizes are set to maximize the cancellation performances, and MULs/DIVs denotes the number of multiplications and divisions to estimate the FIR filters. In the LS, the estimation targets denote the unknown parameters that consist the FIR filters, and in the adaptive algorithm, they denote the FIR filters. For this, the adaptive algorithm has the number of the estimation targets $L^{\prime}$, which is expressed as a function of the impulse response length $L$ and the maximum order to estimate distortions $P$, as much as the LS without the channel of the auxiliary transmitter side. In this paper, the LS solutions such as (32) are calculated by using the LU decomposition, which needs $L^{\prime 3} / 6+L^{\prime 2} / 2-2 L^{\prime} / 3$ 
TABLE III: Cancellation performance and computational complexity

\begin{tabular}{|c|c|c|c|c|c|}
\hline \multicolumn{2}{|c|}{ Estimation algorithms } & Step size & Cancellation $(\mathrm{dB})$ & \# of estimation targets $L^{\prime}$ & MULs/DIVs \\
\hline \hline LS & Prop. $P=5$ & & 60.12 & $(P+1)(P+3) L / 4+2$ & $1.63 \times 10^{8}$ \\
LS & Prop. $P=1$ & & 41.46 & $2 L+2$ & $7.04 \times 10^{6}$ \\
LS & {$[35] P=5$} & & 27.75 & $(P+1) L / 2+1$ & $1.16 \times 10^{7}$ \\
\hline NLMS & Prop. $P=5$ & $7.9 \times 10^{-4}$ & 47.41 & $(P+1)(P+3) L / 4$ & $3.07 \times 10^{6}$ \\
LMS & {$[33]$} & $2.0 \times 10^{-3}$ & 41.52 & $2 L$ & $5.12 \times 10^{5}$ \\
LMS & {$[36] P=5$} & $1.4 \times 10^{-3}$ & 26.95 & $(P+1) L / 2$ & $7.68 \times 10^{5}$ \\
\hline
\end{tabular}

multiplications [55]. For this, when $M$ denotes the number of the received samples in the estimation phase, MULs/DIVs of the $\mathrm{LS}$ is $L^{\prime 3} / 6+(M+3 / 2) L^{\prime 2}+(M+2) L^{\prime}$. Meanwhile, MULs/DIVs of the adaptive algorithm is $L^{\prime} M$. In Table III. the specified value of MULs/DIVs is shown when $M=64000$ and $L=4$.

In the proposed approach at $P=5$, the LS needs about 53 times more MULs/DIVs than the adaptive algorithm. This is because the effects of calculating the LS solution is large. However, the LS can achieve $12.71 \mathrm{~dB}$ higher cancellation performance than the adaptive algorithm. The reason is that the basis functions of different orders $\psi_{p, q}[n]$ are strongly correlated with each other, so that the FIR filter estimation for one basis function is affected by another basis function, and both convergence speed and value of the adaptive algorithm are degraded [36], [54]. Furthermore, the cancellation performances of the proposed approach at $P=1$ and the conventional approach of [33] are equivalent due to the performance restriction by the nonlinear distortion. Similarly, the cancellation performances of the conventional approaches of [35] and [36] are equivalent due to the performance restriction by the IQ imbalance. In this paper, the goal is to cancel as much SI signal as possible in the RF domain and omit the digital SI cancellation. For this reason, it can be found that the proposed approach using the LS, which has the highest cancellation performance, is better than the conventional approaches.

\section{CONCLUSION}

In this paper, to cancel SI present in the RF domain, we have proposed a novel nonlinear SI cancellation approach using the auxiliary transmitter which is effective in the presence of IQ imbalance and nonlinear distortion. The proposed approach estimates the local transceiver channel by using a timedomain LS method and creates the canceling signal based on estimation results and the FIR filter whose coefficients have been derived in this paper. Additionally, we have theoretically derived the SI cancellation limit of the proposed approach. Information about the SI cancellation limit due to phase noise is important for meeting SI cancellation requirements and being able to compare the effects of RF impairments such as IQ imbalance and nonlinear distortion. In simulation results, we have shown that the proposed approach outperforms the conventional approach and the case of using NLMS for the proposed approach. Furthermore, in addition to sharing one LO in the local transceiver, by adjusting the propagation delay of the SI signal and the canceling signal, the SI cancellation limit have been improved.

\section{APPENDiX A}

DERIVATION OF (39)

Using discrete Fourier transformation (DFT), we define this as:

$$
\begin{aligned}
\hat{Y}[k]= & \operatorname{DFT}\{\hat{y}[n]\}, \\
\hat{Y}_{\mathrm{PN}}[k]= & \operatorname{DFT}\left\{\hat{y}_{\mathrm{PN}}[n]\right\} \\
=g_{\mathrm{LNA}} & \left\{\sum_{l=0}^{L-1} \sum_{m=0}^{N-1} h_{\mathrm{tx}}[l] \tilde{X}_{\mathrm{PA}, \mathrm{tx}}[k-m]\right. \\
& \left.\times e^{-j \frac{2 \pi k l}{N}} U_{l}[m]+Z[k]\right\},
\end{aligned}
$$

where $\tilde{X}_{\mathrm{PA}, \mathrm{tx}}[k]=\operatorname{DFT}\left\{\tilde{x}_{\mathrm{PA}, \mathrm{tx}}[n]\right\}, U_{l}[k]=\operatorname{DFT}\left\{u_{l}[n]\right\}$, and $Z[k]=\operatorname{DFT}\left\{z[n] e^{-j \phi\left(n T_{s}\right)}\right\}$. From Parseval's theorem, the power of $\hat{y}[n]$ can be expressed as

$$
\begin{aligned}
\mathbb{E}\left[|\hat{y}[n]|^{2}\right]= & \frac{1}{N^{2}} \sum_{k=0}^{N-1} \mathbb{E}\left[|\hat{Y}[k]|^{2}\right] \\
= & \frac{1}{N^{2}} \sum_{k=0}^{N-1} \mathbb{E}\left[\left|\hat{Y}_{\mathrm{PN}}[k]+\eta_{\mathrm{rx}} \hat{Y}_{\mathrm{PN}}^{*}[-k]\right|^{2}\right] \\
= & \frac{1}{N^{2}} \sum_{k=0}^{N-1} \mathbb{E}\left[\left|\hat{Y}_{\mathrm{PN}}[k]\right|^{2}+\eta_{\mathrm{rx}}^{*} \hat{Y}_{\mathrm{PN}}[k] \hat{Y}_{\mathrm{PN}}[-k]\right. \\
& \left.+\eta_{\mathrm{rx}} \hat{Y}_{\mathrm{PN}}^{*}[-k] \hat{Y}_{\mathrm{PN}}^{*}[k]+\left|\eta_{\mathrm{rx}}\right|^{2}\left|\hat{Y}_{\mathrm{PN}}^{*}[-k]\right|^{2}\right] .
\end{aligned}
$$

In [50, subcarriers are uncorrelated with each other due to symbol mapping, thus $\mathbb{E}\left[\hat{Y}_{\mathrm{PN}}[k] \hat{Y}_{\mathrm{PN}}[-k]\right]=0$. For this, 50 . can be rewritten as

$$
\mathbb{E}\left[|\hat{y}[n]|^{2}\right]=\frac{1}{N^{2}} \sum_{k=0}^{N-1}\left\{\mathbb{E}\left[\left|\hat{Y}_{\mathrm{PN}}[k]\right|^{2}\right]+\left|\eta_{\mathrm{rx}}\right|^{2} \mathbb{E}\left[\left|\hat{Y}_{\mathrm{PN}}[-k]\right|^{2}\right]\right\} .
$$

Furthermore, for (49), the combined phase noise spectrum $\mathbb{E}\left[\left|U_{l}[k]\right|^{2}\right]$ and the spectrum of the PA output characterized by the $\mathrm{AM} / \mathrm{AM}$ and $\mathrm{AM} / \mathrm{PM}$ conversion $\mathbb{E}\left[\left|\tilde{X}_{\mathrm{PA}, \mathrm{tx}}[k]\right|^{2}\right]$ have symmetry. Namely, $\mathbb{E}\left[\left|\hat{Y}_{\mathrm{PN}}[k]\right|^{2}\right]=\mathbb{E}\left[\left|\hat{Y}_{\mathrm{PN}}[-k]\right|^{2}\right]$. Therefore, (51) can be rewritten as

$$
\begin{aligned}
\mathbb{E}\left[|\hat{y}[n]|^{2}\right] & =\left(1+\left|\eta_{\mathrm{rx}}\right|^{2}\right) \frac{1}{N^{2}} \sum_{k=0}^{N-1} \mathbb{E}\left[\left|\hat{Y}_{\mathrm{PN}}[k]\right|^{2}\right] \\
& =\left(1+\left|\eta_{\mathrm{rx}}\right|^{2}\right) \mathbb{E}\left[\left|\hat{y}_{\mathrm{PN}}[n]\right|^{2}\right] .
\end{aligned}
$$




\section{APPENDIX B \\ DERIVATION OF (45)}

From (44), the combined phase noise power is expressed as

$$
\begin{aligned}
\mathbb{E}\left[\left|u_{l}[n]\right|^{2}\right]=\mathbb{E}\left[\left|\left\{e^{j \phi\left((n-l) T_{s}-\tau_{\mathrm{tx}}\right)}-e^{j \phi\left(n T_{s}-\tau_{\text {aux }}\right)}\right\} e^{-j \phi\left(n T_{s}\right)}\right|^{2}\right] \\
=\mathbb{E}\left[\left|e^{j \Phi\left(l T_{s}\right)+j \Phi\left(\tau_{\mathrm{tx}}\right)}-e^{j \Phi\left(\tau_{\text {aux }}\right)}\right|^{2}\right] \\
=\mathbb{E}\left[\left\{e^{j \Phi\left(l T_{s}\right)+j \Phi\left(\tau_{\mathrm{tx}}\right)}-e^{j \Phi\left(\tau_{\mathrm{aux}}\right)}\right\}\right. \\
\left.\quad \times\left\{e^{-j \Phi\left(l T_{s}\right)-j \Phi\left(\tau_{\mathrm{tx}}\right)}-e^{-j \Phi\left(\tau_{\text {aux }}\right)}\right\}\right] \\
=2-\mathbb{E}\left[e^{-j\left\{\Phi\left(\tau_{\text {aux }}\right)-\Phi\left(l T_{s}\right)-\Phi\left(\tau_{\mathrm{tx}}\right)\right\}}\right] \\
\\
\quad-\mathbb{E}\left[e^{j\left\{\Phi\left(\tau_{\text {aux }}\right)-\Phi\left(l T_{s}\right)-\Phi\left(\tau_{\mathrm{tx}}\right)\right\}}\right]
\end{aligned}
$$

For [53, it is found that the phase noise of the receiver does not affect the SI cancellation performance. Here, by (44) and characteristics of Brownian motion, the correlation function of the phase noise can be expressed as [51]

$$
\mathbb{E}\left[e^{j \Phi(\tau)}\right]=e^{-\frac{1}{2} \mathbb{E}\left[|\Phi(\tau)|^{2}\right]}=e^{-2 \pi \beta \tau} .
$$

From (53) and 54, the combined phase noise power can be derived as

$$
\mathbb{E}\left[\left|u_{l}[n]\right|^{2}\right]=2-2 e^{-2 \pi \beta\left|l T_{s}+\tau_{\text {tx }}-\tau_{\text {aux }}\right|} .
$$

\section{REFERENCES}

[1] J. I. Choi, M. Jain, K. Srinivasan, P. Levis, and S. Katti, "Achieving single channel, full duplex wireless communication," in Proc. ACM MobiCom'10, Sept. 2010, pp. 1-12.

[2] A. Aijaz and P. Kulkarni, "Protocol design for enabling full-duplex operation in next-generation IEEE 802.11 WLANs," IEEE Syst. J., vol. 12, no. 4, pp. 3438-3449, Dec. 2018.

[3] M. Kaneko, "Throughput analysis of CSMA with imperfect collision detection in full duplex-enabled WLAN," IEEE Wireless Commun. Lett., vol. 6, no. 4, pp. 490-493, Aug. 2017.

[4] G. Liu, F. R. Yu, H. Ji, V. C. M. Leung, and X. Li, "In-band full-duplex relaying: A survey, research issues and challenges," IEEE Commun. Surveys Tuts., vol. 17, no. 2, pp. 500-524, Jan. 2015.

[5] B. Ma, H. Shah-Mansouri, and V. W. S. Wong, "Full-duplex relaying for D2D communication in millimeter wave-based 5G networks," IEEE Trans. Wireless Commun., vol. 17, no. 7, pp. 4417-4431, Jul. 2018.

[6] E. Hossain and M. Hasan, "5G cellular: key enabling technologies and research challenges," IEEE Instrum. Meas. Mag., vol. 18, no. 3, pp. 11-21, Jun. 2015.

[7] Z. Zhang, X. Chai, K. Long, A. V. Vasilakos, and L. Hanzo, "Full duplex techniques for $5 \mathrm{G}$ networks: self-interference cancellation, protocol design, and relay selection," IEEE Commun. Mag., vol. 53, no. 5, pp. 128-137, May 2015.

[8] R.-A. Pitaval, O. Tirkkonen, R. Wichman, K. Pajukoski, E. Lahetkangas, and E. Tiirola, "Full-duplex self-backhauling for small-cell 5G networks," IEEE Wireless Commu., vol. 22, no. 5, pp. 83-89, Oct. 2015.

[9] A. Sabharwal, P. Schniter, D. Guo, D. W. Bliss, S. Rangarajan, and R. Wichman, "In-band full-duplex wireless: Challenges and opportunities," IEEE J. Sel. Areas in Commu., vol. 32, no. 9, pp. 1637-1652, Sep. 2014.

[10] Z. Zhang, K. Long, A. V. Vasilakos, and L. Hanzo, "Full-duplex wireless communications: Challenges, solutions, and future research directions," Proceedings of the IEEE, vol. 104, no. 7, pp. 1369-1409, Jul. 2016.

[11] J. Zhou, N. Reiskarimian, J. Diakonikolas, T. Dinc, T. Chen, G. Zussman, and H. Krishnaswamy, "Integrated full duplex radios," IEEE Commun. Mag., vol. 55, no. 4, pp. 142-151, Apr. 2017.

[12] K. E. Kolodziej, B. T. Perry, and J. S. Herd, "In-band full-duplex technology: Techniques and systems survey," IEEE Trans. Microw. Theory Tech., pp. 1-17, Feb. 2019.

[13] E. Everett, A. Sahai, and A. Sabharwal, "Passive self-interference suppression for full-duplex infrastructure nodes," IEEE Trans. Wireless Commun., vol. 13, no. 2, pp. 680-694, Feb. 2014.
[14] E. Aryafar, M. A. Khojastepour, K. Sundaresan, S. Rangarajan, and M. Chiang, "MIDU: Enabling mimo full duplex," in Proc. ACM MobiCom'12, ser. Mobicom '12, Aug. 2012, pp. 257-268.

[15] E. Everett, C. Shepard, L. Zhong, and A. Sabharwal, "Softnull: Manyantenna full-duplex wireless via digital beamforming," IEEE Trans. Wireless Commun., vol. 15, no. 12, pp. 8077-8092, Dec. 2016.

[16] E. Manuzzato, J. Tamminen, M. Turunen, D. Korpi, F. Granelli, and M. Valkama, "Digitally-controlled electrical balance duplexer for transmitter-receiver isolation in full-duplex radio," in Proc. 22nd Eur. Wireless Conf., May 2016, pp. 1-8.

[17] L. Laughlin, C. Zhang, M. A. Beach, K. A. Morris, J. L. Haine, M. K. Khan, and M. McCullagh, "Tunable frequency-division duplex RF front end using electrical balance and active cancellation," IEEE Trans. Microw. Theory Tech., vol. 66, no. 12, pp. 5812-5824, Dec. 2018.

[18] T. O'Sullivan, R. A. York, B. Noren, and P. M. Asbeck, "Adaptive duplexer implemented using single-path and multipath feedforward techniques with bst phase shifters," IEEE Trans. Microw. Theory Tech., vol. 53, no. 1, pp. 106-114, Jan. 2005.

[19] B. Debaillie, D. van den Broek, C. Lavïn, B. van Liempd, E. A. M. Klumperink, C. Palacios, J. Craninckx, B. Nauta, and A. Pärssinen, "Analog/RF solutions enabling compact full-duplex radios," IEEE J. Sel. Areas Commun., vol. 32, no. 9, pp. 1662-1673, Sep. 2014.

[20] Y.-S. Choi and H. Shirani-Mehr, "Simultaneous transmission and reception: Algorithm, design and system level performance," IEEE Trans. Wireless Commun., vol. 12, no. 12, pp. 5992-6010, Dec. 2013.

[21] A. Raghavan, E. Gebara, E. M. Tentzeris, and J. Laskar, "Analysis and design of an interference canceller for collocated radios," IEEE Trans. Microw. Theory Tech., vol. 53, no. 11, pp. 3498-3508, Nov. 2005.

[22] T. Huusari, Y. Choi, P. Liikkanen, D. Korpi, S. Talwar, and M. Valkama, "Wideband self-adaptive RF cancellation circuit for full-duplex radio: Operating principle and measurements," in Proc. IEEE 81st Veh. Technol. Conf. (VTC-Spring), May 2015, pp. 1-7.

[23] K. E. Kolodziej, J. G. McMichael, and B. T. Perry, "Multitap RF canceller for in-band full-duplex wireless communications," IEEE Trans. Wireless Commun., vol. 15, no. 6, pp. 4321-4334, Jun. 2016.

[24] Han Su, G. Szczepkowski, and R. Farrell, "Wideband tx leakage cancellation using adaptive delay filter at RF frequencies," in Proc. Irish Signals \& Systems Conference and China-Ireland International Conference on Information and Communications Technologies, Jun. 2014, pp. 396-401.

[25] M. Jain, J. I. Choi, T. Kim, D. Bharadia, S. Seth, K. Srinivasan, P. Levis, S. Katti, and P. Sinha, "Practical, real-time, full duplex wireless," in Proc. ACM MobiCom'11, Sep. 2011, pp. 301-312.

[26] M. Duarte and A. Sabharwal, "Full-duplex wireless communications using off-the-shelf radios: Feasibility and first results," in Proc. Asilomar Conf. Signals Syst. Comput., Nov. 2010, pp. 1558-1562.

[27] M. Duarte, C. Dick, and A. Sabharwal, "Experiment-driven characterization of full-duplex wireless systems," IEEE Trans. Wireless Commun., vol. 11, no. 12, pp. 4296-4307, Dec. 2012.

[28] R. Askar, T. Kaiser, B. Schubert, T. Haustein, and W. Keusgen, "Active self-interference cancellation mechanism for full-duplex wireless transceivers," in 2014 9th International Conference on Cognitive Radio Oriented Wireless Networks and Communications (CROWNCOM), Jun. 2014, pp. 539-544.

[29] J.-H. Lee, "Self-interference cancelation using phase rotation in fullduplex wireless," IEEE Trans. Veh. Technol., vol. 62, no. 9, pp. 44214429, Nov. 2013.

[30] J.-H. Lee, J. won Choi, J.-H. Jung, S.-C. Kim, and Y.-H. Kim, "Analog cancellation for full-duplex wireless in multipath self-interference channels," IEICE Trans. Commun., vol. E98.B, no. 4, pp. 646-652, Apr. 2015.

[31] S. Narieda, "Dual stage analog cancellation of linear and nonlinear self interference for full duplex radio," in 2015 IEEE 26th Annual International Symposium on Personal, Indoor, and Mobile Radio Communications (PIMRC), Aug. 2015, pp. 497-501.

[32] M. Sakai, H. Lin, and K. Yamashita, "Adaptive cancellation of selfinterference in full-duplex wireless with transmitter IQ imbalance," in Proc. IEEE GLOBECOM, Dec. 2014, pp. 3220-3224.

[33] — , "Self-interference cancellation in full-duplex wireless with IQ imbalance," Elsevier Phy. Commun., vol. 18, pp. 2-14, Mar. 2016.

[34] Y. Liu, X. Quan, W. Pan, and Y. Tang, "Digitally assisted analog interference cancellation for in-band full-duplex radios," IEEE Commun. Lett., vol. 21, no. 5, pp. 1079-1082, May 2017.

[35] A. Kiayani, L. Anttila, and M. Valkama, "Active RF cancellation of nonlinear TX leakage in FDD transceivers," in Proc. IEEE Global Conference on Signal and Information Processing (GlobalSIP), Dec. 2016, pp. 689-693. 
[36] A. Kiayani, M. Z. Waheed, L. Anttila, M. Abdelaziz, D. Korpi, V. Syrjälä, M. Kosunen, K. Stadius, J. Ryynänen, and M. Valkama, "Adaptive nonlinear RF cancellation for improved isolation in simultaneous transmit-receive systems," IEEE Trans. Microw. Theory Tech., vol. 66, no. 5, pp. 2299-2312, May 2018.

[37] D. Korpi, L. Anttila, V. Syrjälä, and M. Valkama, "Widely linear digital self-interference cancellation in direct-conversion full-duplex transceiver," IEEE J. Sel. Areas Commun., vol. 32, no. 9, pp. 1674 1687, Sep. 2014.

[38] K. Komatsu, Y. Miyaji, and H. Uehara, "Basis function selection of frequency-domain Hammerstein self-interference canceller for in-band full-duplex wireless communications," IEEE Trans. Wireless Commun., vol. 17 , no. 6, pp. 3768-3780, Jun. 2018.

[39] E. Ahmed, A. M. Eltawil, and A. Sabharwal, "Self-interference cancellation with nonlinear distortion suppression for full-duplex systems," in Proc. 47th Asilomar Conf. Signals, Syst., Comput., Nov. 2013, pp. 1199-1203.

[40] — , "Self-interference cancellation with phase noise induced ICI suppression for full-duplex systems," in Proc. IEEE GLOBECOM, Dec. 2013, pp. 3384-3388.

[41] E. Ahmed and A. M. Eltawil, "On phase noise suppression in full-duplex systems," IEEE Trans. Wireless Commun., vol. 14, no. 3, pp. 1237-1251, Mar. 2015.

[42] — , "All-digital self-interference cancellation technique for full-duplex systems," IEEE Trans. Wireless Commun., vol. 14, no. 7, pp. 3519-3532, Jul. 2015.

[43] V. Syrjälä, M. Valkama, L. Anttila, T. Riihonen, and D. Korpi, "Analysis of oscillator phase-noise effects on self-interference cancellation in fullduplex OFDM radio transceivers," IEEE Trans. Wireless Commun., vol. 13, no. 6, pp. 2977-2990, Jun. 2014.

[44] L. Samara, M. Mokhtar, Ö. Özdemir, R. Hamila, and T. Khattab, "Residual self-interference analysis for full-duplex OFDM transceivers under phase noise and I/Q imbalance," IEEE Commun. Lett., vol. 21, no. 2, pp. 314-317, Feb. 2017.

[45] C. Zhang, L. Laughlin, M. A. Beach, K. A. Morris, and J. L. Haine, "Phase noise in full-duplex radios using off-the-shelf oscillators," IEEE Wireless Commun. Lett., vol. 7, no. 2, pp. 206-209, Apr. 2018.

[46] T. Zhang, C. Su, A. Najafi, and J. C. Rudell, "Wideband dualinjection path self-interference cancellation architecture for full-duplex transceivers," IEEE J. Solid-State Circuits, vol. 53, no. 6, pp. 1563-1576, Jun. 2018

[47] J. Tubbax, B. Come, L. Van der Perre, S. Donnay, M. Engels, Hugo De Man, and M. Moonen, "Compensation of IQ imbalance and phase noise in OFDM systems," IEEE Trans. Wireless Commun., vol. 4, no. 3, pp. 872-877, May 2005.

[48] Q. Zou, M. Mikhemar, and A. H. Sayed, "Digital compensation of crossmodulation distortion in software-defined radios," IEEE J. Sel. Topics Signal Process., vol. 3, no. 3, pp. 348-361, Jun. 2009.

[49] S. Benedetto and E. Biglieri, Principles of Digital Transmission: With Wireless Applications. Norwell, MA, USA: Kluwer Academic Publishers, 1999

[50] P. Banelli and S. Cacopardi, "Theoretical analysis and performance of OFDM signals in nonlinear AWGN channels," IEEE Trans. Commun., vol. 48, no. 3, pp. 430-441, Mar. 2000.

[51] S. Wu and Y. Bar-Ness, "OFDM systems in the presence of phase noise: consequences and solutions," IEEE Trans. Commun., vol. 52, no. 11, pp. 1988-1996, Nov. 2004.

[52] L. Irio and R. Oliveira, "On the impact of fading on residual selfinterference power of in-band full-duplex wireless systems," in 2018 14th International Wireless Communications Mobile Computing Conference (IWCMC), Jun. 2018, pp. 142-146.

[53] C. Rapp, "Effects of HPA-nonlinearity on a 4-DPSK/OFDM-signal for a digital sound broadcasting system," Proc. 2nd European Conf. Satellite Communications, Oct. 1991, pp. 179-184.

[54] D. Korpi, Y.-S. Choi, T. Huusari, L. Anttila, S. Talwar, and M. Valkama, "Adaptive nonlinear digital self-interference cancellation for mobile inband full-duplex radio: Algorithms and RF measurements," in Proc. IEEE GLOBECOM, Dec. 2015, pp. 1-7.

[55] R. Hunger, "Floating point operations in matrix-vector calculus," Technische Universität München, Associate Institute for Signal Processing, Tech. Rep., 2007.

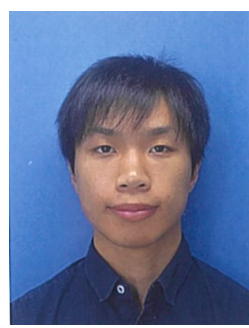

Takahisa Fukui was born in Hokkaido, Japan, on October 8, in 1995. He received B.E. and M.E. degrees from Toyohashi University of Technology, Japan, in 2018 and 2020, respectively. He is currently a researcher in Research and Development BU Sharp Corporation, Chiba, Japan.

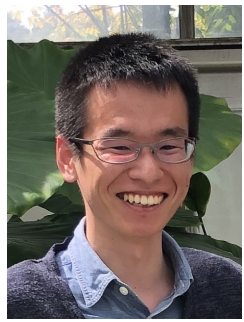

Kazuki Komatsu (S'17) was born in Nara, Japan, in 1994. He received B.E. and M.E. degrees from Toyohashi University of Technology, Japan, in 2016 and 2018 , respectively. He is currently a doctoral student in the Graduate School of Engineering, Toyohashi University of Technology. Since 2019, he has been a Research Fellowship for Young Scientists of the Japan Society for the Promotion of Science (JSPSDC2). His current research interests include in-band full-duplex communications. $\mathrm{He}$ is a member of the IEICE. He received the IEEE Nagoya Section Excellent Student Award in 2016 and the IEEE Communications Society Young Professionals Best Paper Award in 2017.

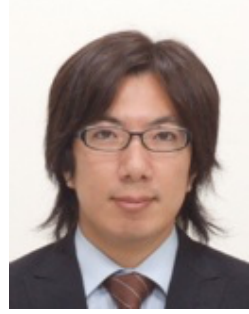

Yuichi Miyaji (S'10-M'13) was born in Kagoshima, Japan, in 1984. He received B.E., M.E., and Ph.D. degrees from Toyohashi University of Technology, Japan, in 2007, 2009, and 2013, respectively. Since 2013, he has been an Assistant Professor at the Department of Electrical and Electronic Information Engineering, Toyohashi University of Technology. His current research interests include wireless networks and in-band full-duplex communications. He is a member of the IEICE and IPSJ. He received the IEICE Young Researcher's Award in 2016 and the IEICE Communications Society: Distinguished Contributions Award in 2016 and 2019

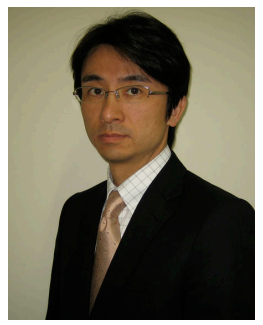

Hideyuki Uehara (S'92-M'97) received B.E., M.E., and $\mathrm{Ph} . \mathrm{D}$. degrees in electrical engineering from Keio University, Yokohama, Japan, in 1992, 1994, and 1997, respectively. Since 1997, he has been with Toyohashi University of Technology, Japan, where he is currently a Professor at the Department of Electrical and Electronic Information Engineering. From 2002 to 2003, he was a Visiting Researcher with the Advanced Telecommunications Research Institute International (ATR), Kyoto, Japan. His current research interests include wireless access and multihop communications. He is a senior member of the IEICE. He received the Distinguished Contributions Award from the IEICE Communications Society in 2002, 2006, 2011, 2012, and 2016. He served as an Editor of the Editorial Committee for the IEICE Transactions on Communications from 2010 to 2011 and a Vice-Chair of the Editorial Board for the IEICE Communications Society from 2014 to 2015. From 2017 to 2018, he served as a Treasurer of the IEEE Nagoya Section. 